\title{
AN APPROXIMATE INVERSE SYSTEM APPROACH TO SHAPE FIBRATIONS
}

\author{
TAKAHISA MiYATA
}

Kobe University, Japan

\begin{abstract}
The notion of shape fibration between compact metric spaces was introduced by S. Mardešić and T. B. Rushing. Mardešić extended the notion to arbitrary topological spaces. A shape fibration $f: X \rightarrow Y$ between topological spaces is defined by using the notion of resolution $(\boldsymbol{p}, \boldsymbol{q}, \boldsymbol{f})$ of the map $f$, where $\boldsymbol{p}: X \rightarrow \boldsymbol{X}$ and $\boldsymbol{q}: Y \rightarrow \boldsymbol{Y}$ are polyhedral resolutions of $X$ and $Y$, respectively, and the approximate homotopy lifting property for the system map $\boldsymbol{f}: \boldsymbol{X} \rightarrow \boldsymbol{Y}$. Although any map $f: X \rightarrow Y$ between topological spaces admits a resolution $(\boldsymbol{p}, \boldsymbol{q}, \boldsymbol{f})$, if polyhedral resolutions $\boldsymbol{p}: X \rightarrow \boldsymbol{X}$ and $\boldsymbol{q}: Y \rightarrow \boldsymbol{Y}$ are chosen in advance, there may not exist a system map $\boldsymbol{f}: \boldsymbol{X} \rightarrow \boldsymbol{Y}$ so that $(\boldsymbol{p}, \boldsymbol{q}, \boldsymbol{f})$ is a resolution of $f$. To overcome this deficiency, T. Watanabe introduced the notion of approximate resolution. An approximate resolution of a map $f: X \rightarrow Y$ consists of approximate polyhedral resolutions $\boldsymbol{p}: X \rightarrow \mathfrak{X}$ and $\boldsymbol{q}: Y \rightarrow \mathfrak{Y}$ of $X$ and $Y$, respectively, and an approximate map $\boldsymbol{f}: \mathfrak{X} \rightarrow \mathfrak{Y}$. In this paper we obtain the approximate homotopy lifting property for approximate maps and investigate its properties. Moreover, it is shown that the approximate homotopy lifting property is extended to the approximate pro-category and the approximate shape category in the sense of Watanabe. It is also shown that the approximate pro-category together with fibrations defined as morphisms having the approximate homotopy lifting property with respect to arbitrary spaces and weak equivalences defined as morphisms inducing isomorphisms in the pro-homotopy category satisfies the composition axiom for a fibration category in the sense of H. J. Baues. As an application it is shown that shape fibrations can be defined in terms of our approximate homotopy lifting property for approximate maps and that every homeomorphism is a shape fibration.
\end{abstract}

2000 Mathematics Subject Classification. 54C56, 55P55.

Key words and phrases. Approximate inverse system, shape fibration, approximate shape, fibration category. 


\section{INTRODUCTION}

The notion of shape fibration between compact metric spaces was first introduced by Mardešić and Rushing ([6]), and it was extended to arbitrary topological spaces by Mardešić ([4]) (see also Q. Haxhibeqiri ([2])). There have been many contributions to the theory of shape fibrations. For a survey of the theory of shape fibrations, see [7, 13].

A shape fibration $f: X \rightarrow Y$ between spaces is a map which admits a resolution $(\boldsymbol{p}, \boldsymbol{q}, \boldsymbol{f})$ of $f$ such that the system map $\boldsymbol{f}: \boldsymbol{X} \rightarrow \boldsymbol{Y}$ between inverse systems has the approximate homotopy lifting property with respect to any space. A resolution $(\boldsymbol{p}, \boldsymbol{q}, \boldsymbol{f})$ of $f$ consists of APol-resolutions $\boldsymbol{p}: X \rightarrow \boldsymbol{X}$ and $\boldsymbol{q}: Y \rightarrow \boldsymbol{Y}$ of $X$ and $Y$, respectively, and a system map $\boldsymbol{f}: \boldsymbol{X} \rightarrow \boldsymbol{Y}$. Here APol denotes the class of approximate polyhedra. A resolution $\boldsymbol{p}=\left(p_{\lambda}\right)$ : $X \rightarrow \boldsymbol{X}$ of a space $X$ consists of an inverse system $\boldsymbol{X}=\left(X_{\lambda}, p_{\lambda \lambda^{\prime}}, \Lambda\right)$ and maps $p_{\lambda}: X \rightarrow X_{\lambda}, \lambda \in \Lambda$, such that $p_{\lambda \lambda^{\prime}} p_{\lambda^{\prime}}=p_{\lambda}$ for $\lambda<\lambda^{\prime}$, and the following two conditions hold:

(i) for any map $h: X \rightarrow P$ into an ANR $P$ and for any open covering $\mathcal{V}$ of $P$, there exist $\lambda \in \Lambda$ and a map $g: X_{\lambda} \rightarrow P$ such that $g p_{\lambda}$ and $h$ are $\mathcal{V}$-near, and

(ii) for any ANR $P$ and for any open covering $\mathcal{V}$ of $P$, there exists an open covering $\mathcal{V}^{\prime}$ of $P$ such that whenever $\lambda \in \Lambda$ and $g, g^{\prime}: X_{\lambda} \rightarrow P$ are maps such that $g p_{\lambda}$ and $g^{\prime} p_{\lambda}$ are $\mathcal{V}^{\prime}$-near, then there exists $\lambda^{\prime}>\lambda$ such that $g p_{\lambda \lambda^{\prime}}$ and $g^{\prime} p_{\lambda \lambda^{\prime}}$ are $\mathcal{V}$-near.

For any class $\mathcal{C}$ of spaces, a $\mathcal{C}$-resolution $\boldsymbol{p}: X \rightarrow \boldsymbol{X}$ means a resolution such that all coordinate spaces of $\boldsymbol{X}$ are in $\mathcal{C}$.

A system map $\boldsymbol{f}=\left(f, f_{\mu}\right): \boldsymbol{X} \rightarrow \boldsymbol{Y}$ between inverse sytems $\boldsymbol{X}$ and $\boldsymbol{Y}=\left(Y_{\mu}, q_{\mu \mu^{\prime}}, M\right)$ consists of a function $f: M \rightarrow \Lambda$ and maps $f_{\mu}: X_{f(\mu)} \rightarrow Y_{\mu}$ for $\mu \in M$ such that for $\mu<\mu^{\prime}$ there exits $\lambda>f(\mu), f\left(\mu^{\prime}\right)$ such that

(M) $f_{\mu} p_{f(\mu) \lambda}=q_{\mu \mu^{\prime}} f_{\mu^{\prime}} p_{f\left(\mu^{\prime}\right) \lambda}$.

The triple $(\boldsymbol{p}, \boldsymbol{q}, \boldsymbol{f})$ is a resolution of $f$ if it satisfies the following condition.

(LM) $f_{\mu} p_{f(\mu)}=q_{\mu} f$ for $\mu \in M$.

However, the theory of resolutions has some defects. Although any map $f$ : $X \rightarrow Y$ admits a resolution $(\boldsymbol{p}, \boldsymbol{q}, \boldsymbol{f})$ of $f$ for some APol-resolutions $\boldsymbol{p}: X \rightarrow \boldsymbol{X}$ and $\boldsymbol{q}: Y \rightarrow \boldsymbol{Y}$ of $X$ and $Y$, respectively, if one chooses resolutions $\boldsymbol{p}$ and $\boldsymbol{q}$ in advance, then there may not exist a system map $\boldsymbol{f}[3,9,11,12]$ (even if $\boldsymbol{X}$ and $\boldsymbol{Y}$ are inverse sytems of compact polyhedra). To overcome this defect, Watanabe [12] introduced the notion of approximate resolution of a space and also of a map.

An approximate inverse system $\mathfrak{X}=\left(X_{\lambda}, \mathcal{U}_{\lambda}, p_{\lambda \lambda^{\prime}}, \Lambda\right)$ consists of an inverse system $\left(X_{\lambda}, p_{\lambda \lambda^{\prime}}, \Lambda\right)$ and an open covering $\mathcal{U}_{\lambda}$ of $X_{\lambda}$, called a mesh, for each $\lambda \in \Lambda$. An approximate map $\boldsymbol{f}=\left(f, f_{\mu}\right): \mathfrak{X} \rightarrow \mathfrak{Y}$ between approximate inverse sytems $\mathfrak{X}$ and $\mathfrak{Y}=\left(Y_{\mu}, \mathcal{V}_{\mu}, q_{\mu \mu^{\prime}}, M\right)$ consists of a function $f: M \rightarrow \Lambda$ 
and maps $f_{\mu}: X_{f(\mu)} \rightarrow Y_{\mu}$, which satisfy a weaker condition expressing approximate commutativity: for $\mu<\mu^{\prime}$, there exits $\lambda>f(\mu), f\left(\mu^{\prime}\right)$ such that

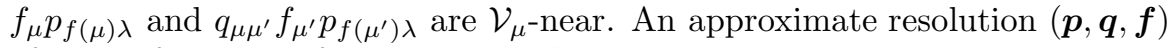
of a map $f$ consists of approximate APol-resolutions $\boldsymbol{p}: X \rightarrow \mathfrak{X}$ and $\boldsymbol{q}: Y \rightarrow \mathfrak{Y}$ of $X$ and $Y$, respectively, and of an approximate map $f: \mathfrak{X} \rightarrow \mathfrak{Y}$, which satisfies a weaker condition, expressing approximate commutativity instead of condition (LM). The upshot is that for arbitrary approximate resolutions $\boldsymbol{p}: X \rightarrow \mathfrak{X}$ and $\boldsymbol{q}: Y \rightarrow \mathfrak{Y}$, chosen in advance, every map $f: X \rightarrow Y$ admits an approximate map $\boldsymbol{f}: \mathfrak{X} \rightarrow \mathfrak{Y}$ so that $(\boldsymbol{p}, \boldsymbol{q}, \boldsymbol{f})$ is an approximate resolution of $f$.

In this paper we extend the approximate homotopy lifting property to approximate maps and investigate their properties. Note here that Mardešić and L. Rubin ([5]) also introduced a more general notion of approximate inverse system of compact metric spaces, which does not assume the commutativity $p_{\lambda \lambda^{\prime}} p_{\lambda^{\prime} \lambda^{\prime \prime}}=p_{\lambda \lambda^{\prime \prime}}$, for $\lambda<\lambda^{\prime}<\lambda^{\prime \prime}$, but assumes only approximate commutativity (see also Mardešić and Watanabe ([8]) for more general spaces). However, in this paper, we concentrate on the commutative case.

More precisely, in this paper we define the approximate homotopy lifting property (AHLP) for approximate maps, and extend it to the approximate pro-category which was introduced by Watanabe ([12]) under the name of approximative pro-cateogry (Section 3). The version of approximate procategory for noncommutative approximate inverse systems can be found in [8]. The construction of approximate pro-category is similar to that of procategory, but the morphisms in the category are based on some nearness property of maps. The approximate pro-category consists of approximate inverse systems as objects and morphisms which are obtained by taking equivalence classes of approximate maps, where the equivalence relation is defined by some nearness of maps, while morphisms in the pro-category are equivalence classes of system maps, where the equivalence relation is defined by the equality of maps. We also show that the property coincides with the AHLP in the sense of Mardešić ([3]) if the approximate map satisfies the commutative condition (M).

Next we obtain a simple characterization of the AHLP for approximate maps using the notion of refinement function in the sense of Watanabe ([12]) (Section 4). Then we consider approximate level maps, which are approximate maps $\boldsymbol{f}=\left(f, f_{\lambda}\right): \mathfrak{X} \rightarrow \mathfrak{Y}$ between approximate inverse systems with the same index set such that the function $f$ is the identity. We simplify the AHLP for approximate level maps.

We show that the approximate pro-category together with fibrations defined as morphisms having the AHLP with respect to any space, and weak equivalences defined as morphisms inducing isomorphisms in the prohomotopy category, satisfies the composition axiom for a fibration category 
in the sense of Baues ([1]) (Section 5). In particular, we show that the composite of morphisms with the AHLP in the approximate pro-category has the AHLP, and that isomorphisms in the approximate pro-category have the AHLP with respect to any space. Using this result, we extend the AHLP to the approximate shape category in the sense of Watanabe $([12])$. The approximate shape category is a category consisting of spaces as objects and of morphisms based on approximate maps, so that the approximate shape category restricted to topologically complete spaces is equivalent to the category of topologically complete spaces and maps. This is a useful category because, in order to study the properties of a space $X$, one can express $X$ as an approximate resolution $\boldsymbol{p}: X \rightarrow \mathfrak{X}$ with an approximate inverse system consisting of polyhedra or ANR's and investigate the appropriate properties of the approximate inverse system.

Finally, we show that the notion of shape fibration is characterized in terms of the AHLP for approximate maps, and as a result we show that every homeomorphism is a shape fibration (Section 6).

\section{Approximate PRO-CATEGORY AND APPROXIMATE SHAPE}

Throughout the paper, a space means a topological space, and a map means a continuous map, unless otherwise stated. Let Top denote the category of spaces and maps.

For any space $X$, let $\operatorname{Cov}(X)$ denote the set of all normal open coverings of $X$. For any subset $A$ of $X$ and $\mathcal{U} \in \operatorname{Cov}(X)$, let $\operatorname{st}(A, \mathcal{U})=\cup\{U \in \mathcal{U}: U \cap A \neq$ $\emptyset\}$. For each $\mathcal{U} \in \operatorname{Cov}(X)$, let $\operatorname{st} \mathcal{U}=\{\operatorname{st}(U, \mathcal{U}): U \in \mathcal{U}\}$. Let $\operatorname{st}^{1} \mathcal{U}=\operatorname{st}_{\mathcal{U}}$ and $\operatorname{st}^{n+1} \mathcal{U}=\operatorname{st}\left(\operatorname{st}^{n} \mathcal{U}\right)$ for each $n=1,2, \ldots$. For any $\mathcal{U} \in \operatorname{Cov}(X)$, two points $x, x^{\prime} \in X$ are $\mathcal{U}$-near, denoted $\left(x, x^{\prime}\right)<\mathcal{U}$, provided $x, x^{\prime} \in U$ for some $U \in \mathcal{U}$. For any $\mathcal{V} \in \operatorname{Cov}(Y)$, two maps $f, g: X \rightarrow Y$ between spaces are $\mathcal{V}$-near, denoted $(f, g)<\mathcal{V}$, provided $(f(x), g(x))<\mathcal{V}$ for each $x \in X$. For each $\mathcal{U} \in \operatorname{Cov}(X)$ and $\mathcal{V} \in \operatorname{Cov}(Y)$, let $f \mathcal{U}=\{f(U): U \in \mathcal{U}\}$ and $f^{-1} \mathcal{V}=\left\{f^{-1}(V): V \in \mathcal{V}\right\}$. For any set $X$, let $1_{X}: X \rightarrow X$ denote the identity function on $X$. For any $\mathcal{U}, \mathcal{U}^{\prime} \in \operatorname{Cov}(X), \mathcal{U}$ is said to refine $\mathcal{U}^{\prime}$, denoted $\mathcal{U}<\mathcal{U}^{\prime}$, provided each $U \in \mathcal{U}^{\prime}$ admits $U^{\prime} \in \mathcal{U}^{\prime}$ such that $U \subseteq U^{\prime}$.

2.1. Approximate systems. An approximate inverse system (approximate system, in short) $\mathfrak{X}=\left(X_{\lambda}, \mathcal{U}_{\lambda}, p_{\lambda \lambda^{\prime}}, \Lambda\right)$ consists of

(1.) a directed preordered set $\Lambda=(\Lambda,<)$ with no maximal element;

(2.) spaces $X_{\lambda}$, for $\lambda \in \Lambda$;

(3.) $\mathcal{U}_{\lambda} \in \operatorname{Cov}\left(X_{\lambda}\right)$, for $\lambda \in \Lambda$; and

(4.) maps $p_{\lambda \lambda^{\prime}}: X_{\lambda^{\prime}} \rightarrow X_{\lambda}$, for $\lambda<\lambda^{\prime}$, such that $p_{\lambda \lambda^{\prime}} p_{\lambda^{\prime} \lambda^{\prime \prime}}=p_{\lambda \lambda^{\prime \prime}}$, for $\lambda<\lambda^{\prime}<\lambda^{\prime \prime}$, and $p_{\lambda \lambda}=1_{X_{\lambda}}$ the identity map on $X_{\lambda}$, for $\lambda \in \Lambda$.

It must satisfy the following two conditions. 
(AI1) For each $\lambda \in \Lambda$ and $\mathcal{U} \in \operatorname{Cov}\left(X_{\lambda}\right)$, there exists $\lambda^{\prime}>\lambda$ such that $\mathcal{U}_{\lambda^{\prime \prime}}<p_{\lambda \lambda^{\prime \prime}}^{-1} \mathcal{U}$ for $\lambda^{\prime \prime}>\lambda^{\prime}$.

(AI2) $\mathcal{U}_{\lambda^{\prime}}<p_{\lambda \lambda^{\prime}}^{-1} \mathcal{U}_{\lambda}$ for $\lambda<\lambda^{\prime}$.

Our definition of approximate system follows that of [12], and our approximate system is a commutative approximate system in the sense of $[5,8]$.

2.2. Approximate resolutions. An approximate map $\boldsymbol{p}=\left(p_{\lambda}\right): X \rightarrow \mathfrak{X}$ of a space $X$ into an approximate system $\mathfrak{X}=\left(X_{\lambda}, \mathcal{U}_{\lambda}, p_{\lambda \lambda^{\prime}}, \Lambda\right)$ consists of maps $p_{\lambda}: X \rightarrow X_{\lambda}$ for $\lambda \in \Lambda$ with the following property.

(AS) For each $\lambda \in \Lambda$ and $\mathcal{U} \in \operatorname{Cov}\left(X_{\lambda}\right)$, there exists $\lambda^{\prime}>\lambda$ such that $\left(p_{\lambda \lambda^{\prime \prime}} p_{\lambda^{\prime \prime}}, p_{\lambda}\right)<\mathcal{U}$ for $\lambda^{\prime \prime}>\lambda^{\prime}$.

An approximate resolution of a space $X$ is an approximate map $\boldsymbol{p}=\left(p_{\lambda}\right)$ : $X \rightarrow \mathfrak{X}$ which satisfies the following two conditions.

(R1) For each ANR $P, \mathcal{V} \in \operatorname{Cov}(P)$ and map $f: X \rightarrow P$, there exist $\lambda \in \Lambda$ and a map $g: X_{\lambda} \rightarrow P$ such that $\left(g p_{\lambda}, f\right)<\mathcal{V}$; and

(R2) For each ANR $P$ and $\mathcal{V} \in \operatorname{Cov}(P)$, there exists $\mathcal{V}^{\prime} \in \operatorname{Cov}(P)$ such that whenever $\lambda \in \Lambda$ and $g, g^{\prime}: X_{\lambda} \rightarrow P$ are maps with $\left(g p_{\lambda}, g^{\prime} p_{\lambda}\right)<$ $\mathcal{V}^{\prime}$, then $\left(g p_{\lambda \lambda^{\prime}}, g^{\prime} p_{\lambda \lambda^{\prime}}\right)<\mathcal{V}$ for some $\lambda^{\prime}>\lambda$.

If $\mathcal{C}$ is a subcategory of spaces, and if all $X_{\lambda}$ are objects in $\mathcal{C}$, then an approximate resolution $\boldsymbol{p}: X \rightarrow \mathfrak{X}$ is called an approximate $\mathcal{C}$-resolution. Here is a useful characterization of an approximate resolution [12, Theorem 3.4].

TheOREM 2.1. An approximate map $\boldsymbol{p}=\left(p_{\lambda}\right): X \rightarrow \mathfrak{X}=\left(X_{\lambda}, \mathcal{U}_{\lambda}, p_{\lambda \lambda^{\prime}}, \Lambda\right)$ is an approximate resolution of a space $X$ if and only if it satisfies the following two conditions.

(B1) For each $\mathcal{U} \in \operatorname{Cov}(X)$, there exists $\lambda \in \Lambda$ such that $p_{\lambda}^{-1} \mathcal{V}<\mathcal{U}$, for some $\mathcal{V} \in \operatorname{Cov}\left(X_{\lambda}\right)$; and

(B2) For each $\lambda \in \Lambda$ and $\mathcal{U} \in \operatorname{Cov}\left(X_{\lambda}\right)$, there exists $\lambda^{\prime}>\lambda$ such that $p_{\lambda \lambda^{\prime}}\left(X_{\lambda^{\prime}}\right) \subseteq \operatorname{st}\left(p_{\lambda}(X), \mathcal{U}\right)$.

Let Pol denote the full subcategory of Top whose objects are polyhedra. Here is an existence theorem for approximate Pol-resolutions [12, Theorem $3.15]$.

TheOREM 2.2. Every space admits an approximate Pol-resolution with a cofinite index set.

2.3. Approximate maps. Let $\mathfrak{X}=\left(X_{\lambda}, \mathcal{U}_{\lambda}, p_{\lambda \lambda^{\prime}}, \Lambda\right)$ and $\mathfrak{Y}=\left(Y_{\mu}, \mathcal{V}_{\mu}, q_{\mu \mu^{\prime}}, M\right)$ be approximate systems of spaces. An approximate map $\boldsymbol{f}=\left(f, f_{\mu}\right): \mathfrak{X} \rightarrow \mathfrak{Y}$ consists of a function $f: M \rightarrow \Lambda$ and maps $f_{\mu}: X_{f(\mu)} \rightarrow Y_{\mu}, \mu \in M$, with the following condition.

(AM) For any $\mu, \mu^{\prime} \in M$ with $\mu<\mu^{\prime}$, there exists $\lambda \in \Lambda$ with $\lambda>$ $f(\mu), f\left(\mu^{\prime}\right)$ such that

$$
\left(q_{\mu \mu^{\prime}} f_{\mu^{\prime}} p_{f\left(\mu^{\prime}\right) \lambda^{\prime}}, f_{\mu} p_{f(\mu) \lambda^{\prime}}\right)<\mathcal{V}_{\mu} \text { for } \lambda^{\prime}>\lambda .
$$


An approximate map $\boldsymbol{f}=\left(f, f_{\mu}\right): \mathfrak{X} \rightarrow \mathfrak{Y}$ is said to be uniform provided it satisfies the following condition.

(U) $\mathcal{U}_{f(\mu)}<f^{-1} \mathcal{V}_{\mu}$ for $\mu \in M$.

It is said to be commutative provided it satisfies the following condition.

(C) For any $\mu, \mu^{\prime} \in M$ with $\mu<\mu^{\prime}$, there exists $\lambda \in \Lambda$ with $\lambda>$ $f(\mu), f\left(\mu^{\prime}\right)$ such that

$$
q_{\mu \mu^{\prime}} f_{\mu^{\prime}} p_{f\left(\mu^{\prime}\right) \lambda^{\prime}}=f_{\mu} p_{f(\mu) \lambda^{\prime}} \text { for } \lambda^{\prime}>\lambda .
$$

A map $f: X \rightarrow Y$ is a limit of an approximate map $\boldsymbol{f}: \mathfrak{X} \rightarrow \mathfrak{Y}$, denoted $\lim \boldsymbol{f}$, provided it satisfies the following condition.

(LAM) For each $\mu \in M$ and $\mathcal{V} \in \operatorname{Cov}\left(Y_{\mu}\right)$, there exists $\mu^{\prime}>\mu$ such that

$$
\left(q_{\mu \mu^{\prime \prime}} f_{\mu^{\prime \prime}} p_{f\left(\mu^{\prime \prime}\right)}, q_{\mu} f\right)<\mathcal{V} \text { for all } \mu^{\prime \prime}>\mu^{\prime} .
$$
$5.8]$.

Here is the existence theorem for limits of approximate maps [8, Theorem

THEOREM 2.3. Let $\boldsymbol{f}: \mathfrak{X} \rightarrow \mathfrak{Y}$ be an approximate map between approximate systems with limits $\boldsymbol{p}: X \rightarrow \mathfrak{X}$ and $\boldsymbol{q}: Y \rightarrow \mathfrak{Y}=\left(Y_{\mu}, \mathcal{V}_{\mu}, q_{\mu \mu^{\prime}}, M\right)$. If all $Y_{\mu}, \mu \in M$, are topologically complete spaces, then $f$ admits a limit map $f=\lim \boldsymbol{f}: X \rightarrow Y$.

For each map $f: X \rightarrow Y$, an approximate resolution of $f$ is a triple $(\boldsymbol{p}, \boldsymbol{q}, \boldsymbol{f})$ consisting of approximate resolutions $\boldsymbol{p}=\left(p_{\lambda}\right): X \rightarrow \mathfrak{X}=$ $\left(X_{\lambda}, \mathcal{U}_{\lambda}, p_{\lambda \lambda^{\prime}}, \Lambda\right)$ and $\boldsymbol{q}=\left(q_{\mu}\right): Y \rightarrow \mathfrak{Y}=\left(Y_{\mu}, \mathcal{V}_{\mu}, q_{\mu \mu^{\prime}}, M\right)$ of $X$ and $Y$, respectively, and of an approximate map $\boldsymbol{f}=\left(f, f_{\mu}\right): \mathfrak{X} \rightarrow \mathfrak{Y}$ with property (LAM).

Let APol denote the full subcategory of Top whose objects are approximate polyhedra (see [4]). Here is the existence theorem for approximate resolutions of any map $[12, \S 4]$.

Theorem 2.4. Let $X$ and $Y$ be spaces. For any approximate resolution $\boldsymbol{p}: X \rightarrow \mathfrak{X}$ of $X$ and for any approximate APol-resolution $\boldsymbol{q}: Y \rightarrow \mathfrak{Y}$ of $Y$ with a cofinite index set, every map $f: X \rightarrow Y$ admits an approximate map $\boldsymbol{f}: \mathfrak{X} \rightarrow \mathfrak{Y}$ such that $(\boldsymbol{p}, \boldsymbol{q}, \boldsymbol{f})$ is an approximate resolution of $f$.

Note here that in Thereom 2.4 the approximate resolutions $\boldsymbol{p}$ and $\boldsymbol{q}$ can be chosen in advance.

2.4. Approximate pro-category. Let $\mathcal{C}$ be any full subcategory of Top. In this subsection we recall the approximate pro-category APRO- $\mathcal{C}$, which was first introduced in [12] under the name of approximative pro-category. For more details, see $[12, \S 2]$. A more general version of the category, whose objects are noncommutative approximate systems as objects, was introduced in [8]. 
For two approximate maps $\boldsymbol{f}=\left(f, f_{\mu}\right), \boldsymbol{f}^{\prime}=\left(f^{\prime}, f_{\mu}^{\prime}\right): \mathfrak{X} \rightarrow \mathfrak{Y}$, we define a relation $\sim$ by putting $\boldsymbol{f} \sim \boldsymbol{f}^{\prime}$ if and only if, for each $\mu \in M$, there exists $\lambda>f(\mu), f^{\prime}(\mu)$ such that

$$
\left(f_{\mu} p_{f(\mu) \lambda}, f_{\mu}^{\prime} p_{f^{\prime}(\mu) \lambda}\right)<\mathcal{V}_{\mu} .
$$

We then define a relation $\equiv$ by putting $f \equiv \boldsymbol{f}^{\prime}$ if and only if there exist finitely many approximate maps $\boldsymbol{f}_{i}: \mathfrak{X} \rightarrow \mathfrak{Y}, i=1,2, \ldots, n$, such that $\boldsymbol{f}=\boldsymbol{f}_{1}$, $\boldsymbol{f}_{i} \sim \boldsymbol{f}_{i+1}, i=1,2, \ldots, n-1, \boldsymbol{f}^{\prime}=\boldsymbol{f}_{n}$. Then the relation $\equiv$ is an equivalence relation, and the equivalence class of $\boldsymbol{f}$ is denoted by $[\boldsymbol{f}]$.

For $n \geq 0$, an increasing function $s: \Lambda \rightarrow \Lambda$ with $s>1_{\Lambda}$ is called an $n$-refinement function of $\mathfrak{X}$ provided $\operatorname{st}^{n} \mathcal{U}_{s(\lambda)}<p_{\lambda s(\lambda)}^{-1} \mathcal{U}_{\lambda}$, for $\lambda \in \Lambda$. For any cofinite index set $\Lambda$ and for each $n \geq 0$, there exists an $n$-refinement function of $\mathfrak{X}$. For any approximate map $\boldsymbol{f}=\left(f, f_{\mu}\right): \mathfrak{X} \rightarrow \mathfrak{Y}$ and for any 1-refinement function of $\mathfrak{Y}$, we have an approximate map $s \boldsymbol{f}=\left(f s, q_{\mu s(\mu)} f_{s(\mu)}\right): X_{f s(\mu)} \rightarrow$ $Y_{\mu}$, which is called the terminal shift of $\boldsymbol{f}$ by $s$, and property (AM) implies the relation $s \boldsymbol{f} \sim \boldsymbol{f}$.

For any full subcategory $\mathcal{C}$ of Top, we define the category APRO- $\mathcal{C}$ as follows. The objects of APRO- $\mathcal{C}$ are approximate systems in $\mathcal{C}$ with cofinite index sets. The set APRO- $\mathcal{C}(\mathfrak{X}, \mathfrak{Y})$ of morphisms $\mathfrak{X} \rightarrow \mathfrak{Y}$ is the set of the equivalence classes of approximate maps $\mathfrak{X} \rightarrow \mathfrak{Y}$ by the equivalence relation $\equiv$. For any $F \in \mathrm{APRO}-\mathcal{C}(\mathfrak{X}, \mathfrak{Y})$ and $G \in \mathrm{APRO}-\mathcal{C}(\mathfrak{Y}, \mathfrak{Z})$, there exist representatives $\boldsymbol{f}$ and $\boldsymbol{g}$ of $F$ and $G$, respectively, which are uniform approximate maps. Then $s(\boldsymbol{g} \boldsymbol{f})=\left(f g s, g_{s(\nu)} f_{g s(\nu)}\right): \mathfrak{X} \rightarrow \mathfrak{Z}=\left(Z_{\nu}, \mathcal{W}_{\nu}, r_{\nu \nu^{\prime}}, N\right)$ is a well-defined approximate map, where $s: N \rightarrow N$ is any 1-refinement function. Let the composite $G F$ be the equivalence class $[s(\boldsymbol{g} \boldsymbol{f})]$. Let the identity $\operatorname{id}_{\mathfrak{X}} \in \mathrm{APRO}-\mathcal{C}(\mathfrak{X}, \mathfrak{X})$ be the equivalence class which is represented by the approximate map $\left(1_{\Lambda}, 1_{X_{\lambda}}\right)$ consisting of the identity function $1_{\Lambda}: \Lambda \rightarrow \Lambda$ and the identity maps $1_{X_{\lambda}}: X_{\lambda} \rightarrow X_{\lambda}$ for $\lambda \in \Lambda$. The objects thus defined and the morphisms together with the composition and the identity form a category, which is denoted by APRO- $\mathcal{C}$ and called the category of approximate systems in $\mathcal{C}$.

For each map $f: X \rightarrow Y$, its approximate resolution $(\boldsymbol{p}, \boldsymbol{q}, \boldsymbol{f})$ is unique in the following sense $[12, \S 4]$.

Theorem 2.5. Let $X$ and $Y$ be spaces. For any approximate resolution $p: X \rightarrow \mathfrak{X}$ of $X$ and for any approximate APol-resolution of $Y$ with cofinite index sets, if $\boldsymbol{f}, \boldsymbol{f}^{\prime}: \mathfrak{X} \rightarrow \mathfrak{Y}$ are approximate maps such that $(\boldsymbol{p}, \boldsymbol{q}, \boldsymbol{f})$ and $\left(\boldsymbol{p}, \boldsymbol{q}, \boldsymbol{f}^{\prime}\right)$ are approximate resolutions of $f$, then $\boldsymbol{f} \equiv \boldsymbol{f}^{\prime}$.

By Theorems 2.4 and 2.5, for arbitrary approximate APol-resolutions $\boldsymbol{p}$ : $X \rightarrow \mathfrak{X}$ and $\boldsymbol{q}: Y \rightarrow \mathfrak{Y}$ with cofinite index sets and for any map $f: X \rightarrow Y$, there exists a unique equivalence class $[\boldsymbol{f}]$ of an approximate map $\boldsymbol{f}: \mathfrak{X} \rightarrow \mathfrak{Y}$ such that $(\boldsymbol{p}, \boldsymbol{q}, \boldsymbol{f})$ is an approximate resolution of $f$. This equivalence class is denoted by $[f]_{p, q}$. 
A pair $(\lambda, \mu) \in \Lambda \times M$ is said to be admissible provided $\mu>f(\lambda)$. For arbitrary admissible pairs $(\lambda, \mu),\left(\lambda^{\prime}, \mu^{\prime}\right) \in \Lambda \times M$, we write $(\lambda, \mu)<\left(\lambda^{\prime}, \mu^{\prime}\right)$ if $\lambda<\lambda^{\prime}$ and $\mu<\mu^{\prime}$.

The following theorem gives a characterization of approximate map inducing an isomorphism in APRO- $\mathcal{C}[12,2.6]$.

THEOREM 2.6. An approximate map $\boldsymbol{f}: \mathfrak{X} \rightarrow \mathfrak{Y}$ induces an isomorphism in APRO- $\mathcal{C}$ if and only if every admissible pair $(\lambda, \mu) \in \Lambda \times M$ admits an admissible pair $\left(\lambda^{\prime}, \mu^{\prime}\right)>(\lambda, \mu)$ and a map $k: Y_{\mu^{\prime}} \rightarrow X_{\lambda}$ such that

(1.) $\left(p_{\lambda \lambda^{\prime}}, k f_{\mu^{\prime}} p_{f\left(\mu^{\prime}\right) \lambda^{\prime}}\right)<\mathcal{U}_{\lambda}$,

(2.) $\mathcal{V}_{\mu^{\prime}}<k^{-1} \mathcal{U}_{\lambda}$

(3.) $\left(q_{\mu \mu^{\prime}}, f_{\mu} p_{f(\mu) \lambda} k\right)<$ st $\mathcal{V}_{\mu}$.

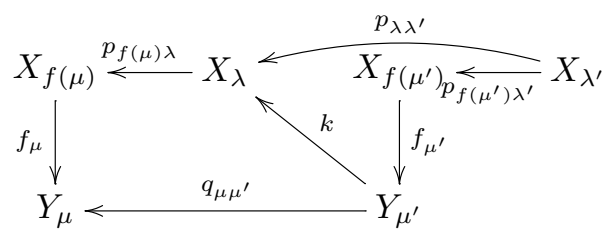

2.5. The approximate shape category. In this subsection we recall the construction of the approximate shape category ASh. For more details, see [12, $\S 7]$.

The objects of ASh are spaces. The set $\operatorname{ASh}(X, Y)$ of morphisms $X \rightarrow Y$ in ASh is defined as follows. Let $E(X)$ denote the set of approximate APolresolutions $\boldsymbol{p}: X \rightarrow \mathfrak{X}$ of $X$ such that $\mathfrak{X}$ has a cofinite index set. For $\boldsymbol{p} \in$ $E(X)$ and $\boldsymbol{q} \in E(Y)$, let $E(\boldsymbol{p}, \boldsymbol{q})=\mathrm{APRO}$-APol $(\mathfrak{X}, \mathfrak{Y})$, where $\boldsymbol{p}: X \rightarrow \mathfrak{X}$ and $\boldsymbol{q}: Y \rightarrow \mathfrak{Y}$. Let $E(X, Y)$ be the disjoint sum of all sets $E(\boldsymbol{p}, \boldsymbol{q})$ for $\boldsymbol{p} \in E(X)$ and $\boldsymbol{q} \in E(Y)$. Then we define a relation $\equiv_{a}$ on the set $E(X, Y)$ by $[\boldsymbol{f}] \equiv_{a}\left[\boldsymbol{f}^{\prime}\right]$ if and only if $\left[\boldsymbol{f}^{\prime}\right]\left[1_{X}\right]_{\boldsymbol{p}, \boldsymbol{p}^{\prime}}=\left[1_{Y}\right]_{\boldsymbol{q}, \boldsymbol{q}^{\prime}}[\boldsymbol{f}]$, where $\boldsymbol{f} \in E(\boldsymbol{p}, \boldsymbol{q})$ and $\boldsymbol{f}^{\prime} \in E\left(\boldsymbol{p}^{\prime}, \boldsymbol{q}^{\prime}\right)$. It is readily seen that this is an equivalence relation on $E(X, Y)$, and the equivalence class of $[\boldsymbol{f}]$ is denoted by $\langle[\boldsymbol{f}]\rangle$. Let $\langle E(X, Y)\rangle=$ $\{\langle[\boldsymbol{f}]\rangle:[\boldsymbol{f}] \in E(X, Y)\}$. Then $\langle E(X, Y)\rangle$ is a set since there is a bijection $\Phi(\boldsymbol{p}, \boldsymbol{q}): E(\boldsymbol{p}, \boldsymbol{q}) \rightarrow\langle E(X, Y)\rangle$ for any $\boldsymbol{p} \in E(X)$ and $\boldsymbol{q} \in E(Y)$ and $E(\boldsymbol{p}, \boldsymbol{q})$ is a set. The set $\operatorname{ASh}(X, Y)$ of morphisms $X \rightarrow Y$ is defined as the set $\langle E(X, Y)\rangle$. For any $\langle[\boldsymbol{f}]\rangle \in \operatorname{ASh}(X, Y)$ and $\langle[\boldsymbol{g}]\rangle \in \operatorname{ASh}(Y, Z)$, we define the composite $\langle[\boldsymbol{g}]\rangle \circ\langle[\boldsymbol{f}]\rangle$ as $\left\langle[\boldsymbol{g}]\left[1_{Y}\right]_{\boldsymbol{q}, \boldsymbol{q}^{\prime}}[\boldsymbol{f}]\right\rangle$, where $\boldsymbol{f} \in E(\boldsymbol{p}, \boldsymbol{q})$ and $\boldsymbol{g} \in E\left(\boldsymbol{q}^{\prime}, \boldsymbol{r}^{\prime}\right)$. Let the identity $\operatorname{id}_{X} \in \operatorname{ASh}(X, X)$ be the morphism $\left\langle\left[1_{X}\right]_{\boldsymbol{p}, \boldsymbol{p}}\right\rangle$. Then ASh together with thus defined composites and identities form a category.

There is a well-defined functor $A S:$ Top $\rightarrow$ ASh which is defined by $A S(X)=X$ for each object $X$ of Top and $A S(f)=\left\langle[f]_{\boldsymbol{p}, \boldsymbol{q}}\right\rangle$ for each map $f: X \rightarrow Y$ and for some fixed $\boldsymbol{p} \in E(X)$ and $\boldsymbol{q} \in E(Y)$.

For any approximate maps $\boldsymbol{f}, \boldsymbol{f}^{\prime}: \mathfrak{X} \rightarrow \mathfrak{Y}$ between topologically complete spaces, $\boldsymbol{f} \sim \boldsymbol{f}^{\prime}$ implies $\lim \boldsymbol{f}=\lim \boldsymbol{f}^{\prime}$. So for each $[\boldsymbol{f}] \in E(\boldsymbol{p}, \boldsymbol{q}), \lim [\boldsymbol{f}]=$ 
$\lim \boldsymbol{f}$ is well-defined. Let $\mathrm{CTop}_{3.5}$ denote the full subcategory of Top whose objects are topologically complete spaces, and let $\mathrm{ASh}\left(\mathrm{CTop}_{3.5}\right)$ denote the restriction of the category ASh to topologically complete spaces. There is a realization functor $R: \mathrm{ASh}\left(\mathrm{CTop}_{3.5}\right) \rightarrow \mathrm{CTop}_{3.5}$ which is defined by $R(X)=$ $X$ for each object $X$ of $\mathrm{CTop}_{3.5}$ and $R(F)=\lim \Phi(\boldsymbol{p}, \boldsymbol{q})^{-1}(F)$ for each $F \in$ $\operatorname{ASh}\left(\mathrm{CTop}_{3.5}\right)(X, Y)$ and for some fixed $\boldsymbol{p} \in E(X)$ and $\boldsymbol{q} \in E(Y)$. Then the following theorem shows that there is an equivalence of categories $\mathrm{CTop}_{3.5}$ and $\mathrm{ASh}\left(\mathrm{CTop}_{3.5}\right)$.

TheOrEm 2.7. $R \circ A S \mid \mathrm{CTop}_{3.5}=1_{\mathrm{CTop}_{3.5}}$ and $A S \mid \mathrm{CTop}_{3.5} \circ R=$ $1_{\mathrm{ASh}\left(\mathrm{CTop}_{3.5}\right)}$.

\section{Approximate homotopy LIFTING PROPERTY}

In this section we define the approximate homotopy lifting property for morphisms in the approximate pro-category APRO- $\mathcal{C}$ for any full subcategory $\mathcal{C}$ of Top, and later in Section 6 we extend it to morphisms in the approximate shape category ASh. First, we define the approximate homotopy lifting property for approximate maps.

An approximate map $\boldsymbol{f}=\left(f, f_{\mu}\right): \mathfrak{X} \rightarrow \mathfrak{Y}$ between approximate systems $\mathfrak{X}=\left(X_{\lambda}, \mathcal{U}_{\lambda}, p_{\lambda \lambda^{\prime}}, \Lambda\right)$ and $\mathfrak{Y}=\left(Y_{\mu}, \mathcal{V}_{\mu}, q_{\mu \mu^{\prime}}, M\right)$ in $\mathcal{C}$ has the approximate homotopy lifting property (AHLP) with respect to a space $Z$ provided it satisfies the following property.

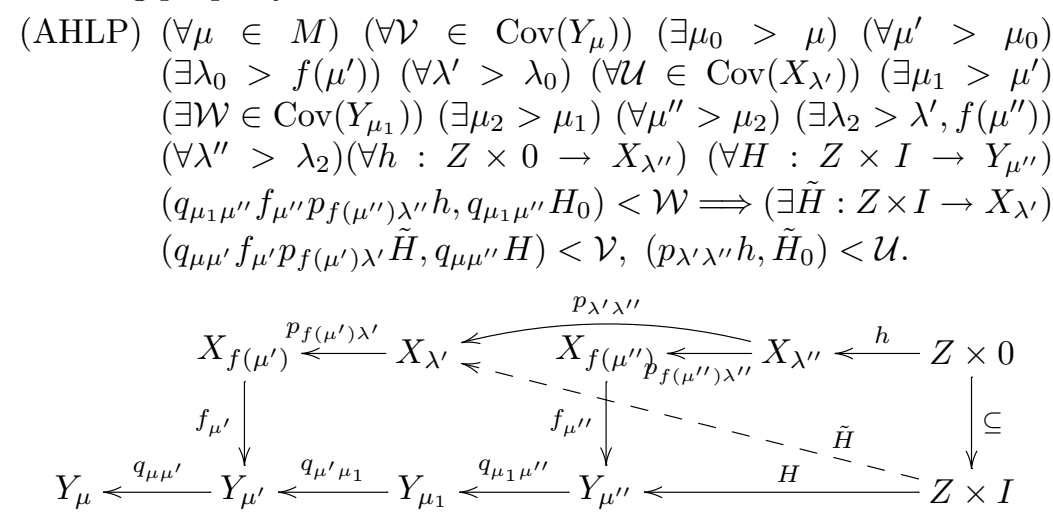

Let $\boldsymbol{f}=\left(f, f_{\mu}\right): \mathfrak{X} \rightarrow \mathfrak{Y}$ be an approximate map between approximate systems $\mathfrak{X}=\left(X_{\lambda}, \mathcal{U}_{\lambda}, p_{\lambda \lambda^{\prime}}, \Lambda\right)$ and $\mathfrak{Y}=\left(Y_{\mu}, \mathcal{V}_{\mu}, q_{\mu \mu^{\prime}}, M\right)$ in $\mathcal{C}$, and suppose that $\boldsymbol{f}$ has the AHLP with respect to a space $Z$. For convenience, we introduce the following notation. For any $\mu \in M$ and $\mathcal{V} \in \operatorname{Cov}\left(Y_{\mu}\right)$, let $\operatorname{AHLP}(\mu, \mathcal{V})$ denote the set of all $\mu_{0} \in M$ such that $\mu_{0}>\mu$ and $\mu_{0}$ satisfies the condition in (AHLP). For any $\mu \in M, \mathcal{V} \in \operatorname{Cov}\left(Y_{\mu}\right)$, and $\mu^{\prime}>\mu_{0}$ where $\mu_{0} \in \operatorname{AHLP}(\mu, \mathcal{V})$, let $\operatorname{AHLP}\left(\mu, \mathcal{V}, \mu^{\prime}\right)$ denote the set of all $\lambda_{0} \in \Lambda$ such that $\lambda_{0}>f\left(\mu^{\prime}\right)$ and $\lambda_{0}$ satisfies the condition in (AHLP). For any $\mu \in M, \mathcal{V} \in \operatorname{Cov}\left(Y_{\mu}\right), \mu^{\prime}>\mu_{0}$ where $\mu_{0} \in \operatorname{AHLP}(\mu, \mathcal{V}), \lambda^{\prime}>\lambda_{0}$ where 
$\lambda_{0} \in \operatorname{AHLP}\left(\mu, \mathcal{V}, \mu^{\prime}\right)$, and $\mathcal{U} \in \operatorname{Cov}\left(X_{\lambda^{\prime}}\right)$, let $\operatorname{AHLP}\left(\mu, \mathcal{V}, \mu^{\prime}, \lambda^{\prime}, \mathcal{U}\right)$ denote the set of all triples $\left(\mu_{1}, \mathcal{W}, \mu_{2}\right) \in M \times \operatorname{Cov}\left(Y_{\mu_{1}}\right) \times M$ such that $\mu_{2}>\mu_{1}>\mu^{\prime}$ and $\mu_{1}, \mu_{2}$, and $\mathcal{W}$ satisfy the condition in (AHLP). For any $\mu \in M, \mathcal{V} \in \operatorname{Cov}\left(Y_{\mu}\right)$, $\mu^{\prime}>\mu_{0}$ where $\mu_{0} \in \operatorname{AHLP}(\mu, \mathcal{V}), \lambda^{\prime}>\lambda_{0}$ where $\lambda_{0} \in \operatorname{AHLP}\left(\mu, \mathcal{V}, \mu^{\prime}\right)$, $\mathcal{U} \in \operatorname{Cov}\left(X_{\lambda^{\prime}}\right)$, and $\mu^{\prime \prime}>\mu_{2}$ where $\left(\mu_{1}, \mathcal{W}, \mu_{2}\right) \in \operatorname{AHLP}\left(\mu, \mathcal{V}, \mu^{\prime}, \lambda^{\prime}\right)$ for some $\mu_{1} \in M$ and $\mathcal{W} \in \operatorname{Cov}\left(Y_{\mu_{1}}\right)$, let $\operatorname{AHLP}\left(\mu, \mathcal{V}, \mu^{\prime}, \lambda^{\prime}, \mathcal{U}, \mu^{\prime \prime}\right)$ denote the set of all $\lambda_{2} \in \Lambda$ such that $\lambda_{2}>\lambda^{\prime}, f\left(\mu^{\prime \prime}\right)$ and $\lambda_{2}$ satisfies the condition in (AHLP).

For commutative approximate maps, our definition of AHLP coincides with that of Mardešić. Recall the approximate homotopy lifting property in the sense of Mardešić (see [4]). A system map $\boldsymbol{f}=\left(f, f_{\mu}\right): \boldsymbol{X} \rightarrow \boldsymbol{Y}$ between systems $\boldsymbol{X}=\left(X_{\lambda}, p_{\lambda \lambda^{\prime}}, \Lambda\right)$ and $\boldsymbol{Y}=\left(Y_{\mu}, q_{\mu \mu^{\prime}}, M\right)$ has the approximate homotopy lifting property (AHLP) with respect to a space $Z$ provided it satisfies the following property.

$(\mathrm{AHLP})_{c}$ For any admissible pair $(\lambda, \mu) \in \Lambda \times M$ and for any $\mathcal{U} \in$ $\operatorname{Cov}\left(X_{\lambda}\right)$ and $\mathcal{V} \in \operatorname{Cov}\left(Y_{\mu}\right)$ there exist an admissible pair $\left(\lambda^{\prime}, \mu^{\prime}\right)>(\lambda, \mu)$ and $\mathcal{V}^{\prime} \in \operatorname{Cov}\left(Y_{\mu^{\prime}}\right)$ such that whenever $h: Z \times 0 \rightarrow X_{\lambda^{\prime}}$ and $H: Z \times I \rightarrow Y_{\mu^{\prime}}$ are maps with $\left(f_{\mu^{\prime}} p_{f\left(\mu^{\prime}\right) \lambda^{\prime}} h, H_{0}\right)<\mathcal{V}^{\prime}$, there exists a map $\tilde{H}: Z \times I \rightarrow X_{\lambda}$ such that $\left(p_{\lambda \lambda^{\prime}} h, \tilde{H}_{0}\right)<\mathcal{U}$, and $\left(f_{\mu} p_{f(\mu) \lambda} \tilde{H}, q_{\mu \mu^{\prime}} H\right)<\mathcal{V}$.

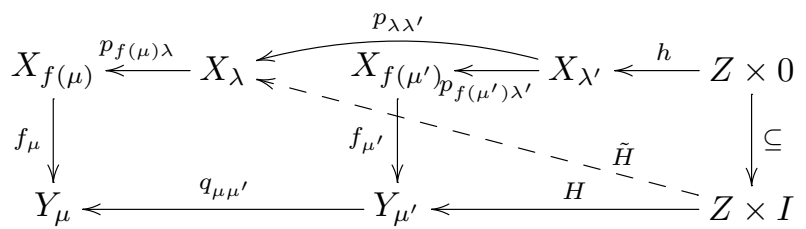

THEOREM 3.1. For any commutative approximate map $\boldsymbol{f}=\left(f, f_{\mu}\right)$ : $\mathfrak{X} \rightarrow \mathfrak{Y}$ between approximate systems $\mathfrak{X}=\left(X_{\lambda}, \mathcal{U}_{\lambda}, p_{\lambda \lambda^{\prime}}, \Lambda\right)$ and $\mathfrak{Y}=$ $\left(Y_{\mu}, \mathcal{V}_{\mu}, q_{\mu \mu^{\prime}}, M\right)$ in $\mathcal{C}$, the approximate map $\boldsymbol{f}$ has property (AHLP) with respect to a space $Z$ if and only if the system map $\boldsymbol{f}=\left(f, f_{\mu}\right): \boldsymbol{X} \rightarrow \boldsymbol{Y}$ between systems $\boldsymbol{X}=\left(X_{\lambda}, p_{\lambda \lambda^{\prime}}, \Lambda\right)$ and $\boldsymbol{Y}=\left(Y_{\mu}, q_{\mu \mu^{\prime}}, M\right)$ has property $(A H L P)_{c}$ with respect to $Z$.

Proof. Suppose that the system map $\left(f, f_{\mu}\right): \boldsymbol{X} \rightarrow \boldsymbol{Y}$ has property $(\mathrm{AHLP})_{c}$ with respect to a space $Z$. Let $\mu \in M$, and let $\mathcal{V} \in \operatorname{Cov}\left(Y_{\mu}\right)$. Set $\mu_{0}=\mu$ and $\lambda_{0}=f\left(\mu_{0}\right)$. Let $\left(\lambda^{\prime}, \mu^{\prime}\right)>\left(\lambda_{0}, \mu_{0}\right)$ be any admissible pair, and let $\mathcal{U} \in \operatorname{Cov}\left(X_{\lambda^{\prime}}\right)$. Apply property $(\mathrm{AHLP})_{c}$ to the admissible pair $\left(\lambda^{\prime}, \mu^{\prime}\right)$ and the open covering $q_{\mu \mu^{\prime}}^{-1} \mathcal{V} \in \operatorname{Cov}\left(Y_{\mu^{\prime}}\right)$, and obtain an admissible pair $\left(\lambda_{1}, \mu_{1}\right) \geq$ $\left(\lambda^{\prime}, \mu^{\prime}\right)$ and $\mathcal{W} \in \operatorname{Cov}\left(Y_{\mu_{1}}\right)$ which satisfy condition $(\mathrm{AHLP})_{c}$. Let $\mu_{2}=\mu_{1}$, and let $\mu^{\prime \prime}>\mu_{2}$. Then by $(\mathrm{C})$ there is $\lambda_{2}>f\left(\mu^{\prime \prime}\right), \lambda_{1}$ such that

$$
f_{\mu_{1}} p_{f\left(\mu_{1}\right) \lambda^{\prime \prime}}=q_{\mu_{1} \mu^{\prime \prime}} f_{\mu^{\prime \prime}} p_{f\left(\mu^{\prime \prime}\right) \lambda^{\prime \prime}} \text { for } \lambda^{\prime \prime}>\lambda_{2} .
$$


Let $\lambda^{\prime \prime}>\lambda_{2}$, and let $h: Z \times 0 \rightarrow X_{\lambda^{\prime \prime}}$ and $H: Z \times I \rightarrow Y_{\mu^{\prime \prime}}$ be maps such that

$$
\left(q_{\mu_{1} \mu^{\prime \prime}} f_{\mu^{\prime \prime}} p_{f\left(\mu^{\prime \prime}\right) \lambda^{\prime \prime}} h, q_{\mu_{1} \mu^{\prime \prime}} H_{0}\right)<\mathcal{W} .
$$

By (3.1) and (3.2),

$$
\left(f_{\mu_{1}} p_{f\left(\mu_{1}\right) \lambda^{\prime \prime}} h, q_{\mu_{1} \mu^{\prime \prime}} H_{0}\right)<\mathcal{W} .
$$

By $(\text { AHLP })_{c}$, there exists a map $\tilde{H}: Z \times I \rightarrow X_{\lambda^{\prime}}$ such that

$$
\begin{array}{r}
\left(p_{\lambda^{\prime} \lambda^{\prime \prime}} h, \tilde{H}_{0}\right)<\mathcal{U}, \\
\left(f_{\mu^{\prime}} p_{f\left(\mu^{\prime}\right) \lambda^{\prime}} \tilde{H}, q_{\mu^{\prime} \mu^{\prime \prime}} H\right)<q_{\mu \mu^{\prime}}^{-1} \mathcal{V} .
\end{array}
$$

The latter implies

$$
\left(q_{\mu \mu^{\prime}} f_{\mu^{\prime}} p_{f\left(\mu^{\prime}\right) \lambda^{\prime}} \tilde{H}, q_{\mu \mu^{\prime \prime}} H\right)<\mathcal{V} .
$$

This shows that the approximate map $\boldsymbol{f}$ has property (AHLP).

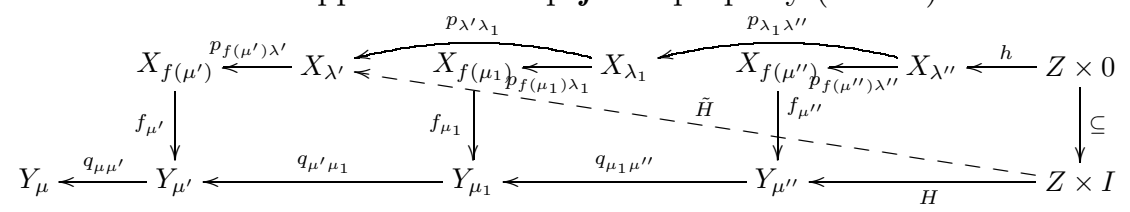

Conversely, suppose that the approximate map $f$ has property (AHLP) with respect to a space $Z$. Let $(\lambda, \mu) \in \Lambda \times M$ be an admissible pair, and let $\mathcal{V} \in \operatorname{Cov}\left(Y_{\mu}\right)$ and $\mathcal{U} \in \operatorname{Cov}\left(X_{\lambda}\right)$. Choose $\mu_{0} \in \operatorname{AHLP}(\mu, \mathcal{V})$, and let $\mu^{\prime}>\mu_{0}$. Choose $\lambda_{0} \in \operatorname{AHLP}\left(\mu, \mathcal{V}, \mu^{\prime}\right)$. Then by (C) there exists $\lambda^{\prime}>\lambda_{0}$ such that

$$
f_{\mu} p_{f(\mu) \lambda^{\prime}}=q_{\mu \mu^{\prime}} f_{\mu^{\prime}} p_{f\left(\mu^{\prime}\right) \lambda^{\prime}} .
$$

Choose a triple $\left(\mu_{1}, \mathcal{W}, \mu_{2}\right) \in \operatorname{AHLP}\left(\mu, \mathcal{V}, \mu^{\prime}, \lambda^{\prime}, p_{\lambda \lambda^{\prime}}^{-1} \mathcal{U}\right)$, and let $\mu^{\prime \prime}>\mu_{2}$. Choose $\lambda_{2} \in \operatorname{AHLP}\left(\mu, \mathcal{V}, \mu^{\prime}, \lambda^{\prime}, p_{\lambda \lambda^{\prime}}^{-1} \mathcal{U}, \mu^{\prime \prime}\right)$, and let $\lambda^{\prime \prime}>\lambda_{2}$. Then the admissible pair $\left(\lambda^{\prime \prime}, \mu^{\prime \prime}\right)$ and the open covering $q_{\mu_{1} \mu^{\prime \prime}}^{-1} \mathcal{W} \in \operatorname{Cov}\left(Y_{\mu^{\prime \prime}}\right)$ satisfy condition $(\mathrm{AHLP})_{c}$. Indeed, suppose that $h: Z \times 0 \rightarrow X_{\lambda^{\prime \prime}}$ and $H: Z \times I \rightarrow Y_{\mu^{\prime \prime}}$ are maps such that

$$
\left(f_{\mu^{\prime \prime}} p_{f\left(\mu^{\prime \prime}\right) \lambda^{\prime \prime}} h, H_{0}\right)<q_{\mu_{1} \mu^{\prime \prime}}^{-1} \mathcal{W} .
$$

Then by property (AHLP), there exists a map $\tilde{H}: Z \times I \rightarrow X_{\lambda^{\prime}}$ such that

$$
\begin{array}{r}
\left(p_{\lambda^{\prime} \lambda^{\prime \prime}} h, \tilde{H}_{0}\right)<p_{\lambda \lambda^{\prime}}^{-1} \mathcal{U}, \\
\left(q_{\mu \mu^{\prime}} f_{\mu^{\prime}} p_{f\left(\mu^{\prime}\right) \lambda^{\prime}} \tilde{H}, q_{\mu \mu^{\prime \prime}} H\right)<\mathcal{V} .
\end{array}
$$

(3.3), (3.4), and (3.5) imply

$$
\begin{aligned}
\left(p_{\lambda \lambda^{\prime \prime}} h, p_{\lambda \lambda^{\prime}} \tilde{H}_{0}\right) & <\mathcal{U}, \\
\left(f_{\mu} p_{f(\mu) \lambda} \tilde{H}, q_{\mu \mu^{\prime \prime}} H\right) & <\mathcal{V},
\end{aligned}
$$

as required. 


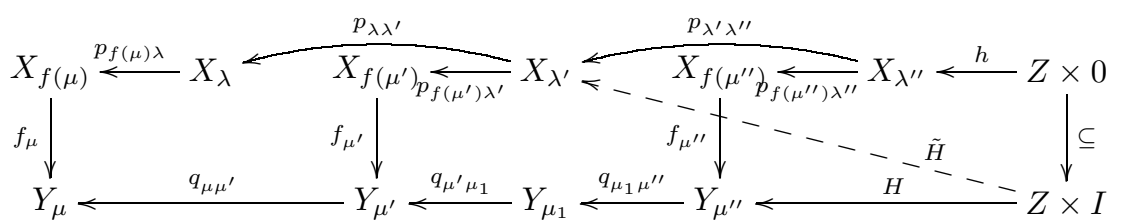

Next, we extend the definition of the AHLP to morphisms in APRO- $\mathcal{C}$.

Theorem 3.2. Let $\boldsymbol{f}=\left(f, f_{\mu}\right), \boldsymbol{g}=\left(g, g_{\mu}\right): \mathfrak{X} \rightarrow \mathfrak{Y}$ be approximate maps. If $\boldsymbol{f} \sim \boldsymbol{g}$ and $\boldsymbol{f}$ has the AHLP with respect to a space $Z$, then so does g.

Proof. Let $\mu \in M$ and $\mathcal{V} \in \operatorname{Cov}\left(Y_{\mu}\right)$. Take $\mathcal{V}^{\prime} \in \operatorname{Cov}\left(Y_{\mu}\right)$ such that

$$
\text { st } \mathcal{V}^{\prime}<\mathcal{V} \text {. }
$$

Choose $\mu_{0} \in \operatorname{AHLP}\left(\mu, \mathcal{V}^{\prime}\right)$ such that

$$
\mathcal{V}^{\prime}<q_{\mu \mu^{\prime}}^{-1} \mathcal{V}_{\mu} \text { for } \mu^{\prime}>\mu_{0}
$$

Let $\mu^{\prime}>\mu_{0}$. By $\boldsymbol{f} \sim \boldsymbol{g}$, we can choose $\lambda_{1} \in \operatorname{AHLP}\left(\mu, \mathcal{V}^{\prime}, \mu^{\prime}\right)$ such that $\lambda_{1}>f(\mu), f\left(\mu^{\prime}\right)$ and

$$
\left(f_{\mu^{\prime}} p_{f\left(\mu^{\prime}\right) \lambda^{\prime}}, g_{\mu^{\prime}} p_{g\left(\mu^{\prime}\right) \lambda^{\prime}}\right)<\mathcal{V}_{\mu^{\prime}} \text { for } \lambda^{\prime}>\lambda_{1} .
$$

Let $\lambda^{\prime}>\lambda_{1}$ and $\mathcal{U} \in \operatorname{Cov}\left(X_{\lambda^{\prime}}\right)$. Choose a triple $\left(\mu_{1}, \mathcal{W}, \mu_{2}\right) \in \operatorname{AHLP}\left(\mu, \mathcal{V}^{\prime}, \mu^{\prime}\right.$, $\left.\lambda^{\prime}, \mathcal{U}\right)$ such that

$$
\mathcal{V}_{\mu^{\prime \prime}}<q_{\mu_{1} \mu^{\prime \prime}}^{-1} \mathcal{W}^{\prime} \text { for } \mu^{\prime \prime}>\mu_{2}
$$

where $\mathcal{W}^{\prime} \in \operatorname{Cov}\left(Y_{\mu_{1}}\right)$ satisfies

$$
\text { st } \mathcal{W}^{\prime}<\mathcal{W} .
$$

Let $\mu^{\prime \prime}>\mu_{2}$. By $\boldsymbol{f} \sim \boldsymbol{g}$, we can choose $\lambda_{2} \in \operatorname{AHLP}\left(\mu, \mathcal{V}^{\prime}, \mu^{\prime}, \lambda^{\prime}, \mathcal{U}, \mu^{\prime \prime}\right)$ such that $\lambda_{2}>\lambda^{\prime}, f\left(\mu^{\prime \prime}\right), g\left(\mu^{\prime \prime}\right)$ and

$$
\left(f_{\mu^{\prime \prime}} p_{f\left(\mu^{\prime \prime}\right) \lambda^{\prime \prime}}, g_{\mu^{\prime \prime}} p_{g\left(\mu^{\prime \prime}\right) \lambda^{\prime \prime}}\right)<\mathcal{V}_{\mu^{\prime \prime}} \text { for } \lambda^{\prime \prime}>\lambda_{2} .
$$

Let $\mu^{\prime \prime}>\lambda_{2}$, and let $h: Z \times 0 \rightarrow X_{\lambda^{\prime \prime}}$ and $H: Z \times I \rightarrow Y_{\mu^{\prime \prime}}$ be maps such that

$$
\left(q_{\mu_{1} \mu^{\prime \prime}} g_{\mu^{\prime \prime}} p_{g\left(\mu^{\prime \prime}\right) \lambda^{\prime \prime}} h, q_{\mu_{1} \mu^{\prime \prime}} H_{0}\right)<\mathcal{W}^{\prime}
$$

Then by (3.9), (3.10), (3.11), (3.12),

$$
\left(q_{\mu_{1} \mu^{\prime \prime}} f_{\mu^{\prime \prime}} p_{f\left(\mu^{\prime \prime}\right) \lambda^{\prime \prime}} h, q_{\mu_{1} \mu^{\prime \prime}} H_{0}\right)<\mathcal{W} .
$$

By property (AHLP) for $\boldsymbol{f}$, there exists a map $\tilde{H}: Z \times I \rightarrow X_{\lambda^{\prime}}$ such that

$$
\begin{aligned}
\left(p_{\lambda^{\prime} \lambda^{\prime \prime}}\right. & \left., \tilde{H}_{0}\right)<\mathcal{U}, \\
\left(q_{\mu \mu^{\prime}} f_{\mu^{\prime}} p_{f\left(\mu^{\prime}\right) \lambda^{\prime}} \tilde{H}, q_{\mu \mu^{\prime \prime}} H\right) & <\mathcal{V}^{\prime} .
\end{aligned}
$$


So, by (3.6), (3.7), (3.8), (3.14),

$$
\left(q_{\mu \mu^{\prime}} g_{\mu^{\prime}} p_{g\left(\mu^{\prime}\right) \lambda^{\prime}} \tilde{H}, q_{\mu \mu^{\prime \prime}} H\right)<\mathcal{V} .
$$

By (3.13) and (3.15), we conclude that $\boldsymbol{g}$ has the AHLP.

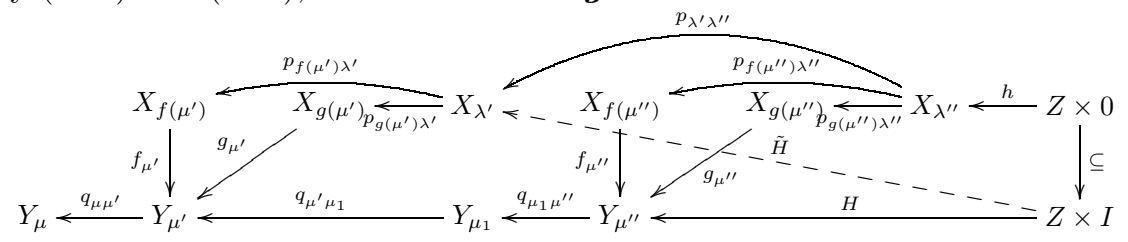

A morphism $F: \mathfrak{X} \rightarrow \mathfrak{Y}$ in APRO- $\mathcal{C}$ is said to have the approximate lifting property (AHLP) with respect to a space $Z$ provided some representative $\boldsymbol{f}$ of $F$ has the AHLP with respect to $Z$. By Theorem 3.2 this is equivalent to saying that every representative $f$ of $F$ has the AHLP with respect to $Z$.

\section{Properties (AhLP)* And (AHLP $)_{L}$}

For any approximate map $\boldsymbol{f}=\left(f, f_{\mu}\right): \mathfrak{X} \rightarrow \mathfrak{Y}$ and for any space $Z$, consider the following property.

(AHLP)* For any admissible pair $(\lambda, \mu) \in \Lambda \times M$, there exists an admissible pair $\left(\lambda^{\prime}, \mu^{\prime}\right)>(\lambda, \mu)$ such that whenever $h$ : $Z \times 0 \rightarrow X_{\lambda^{\prime}}$ and $H: Z \times I \rightarrow Y_{\mu^{\prime}}$ are maps such that $\left(H_{0}, f_{\mu^{\prime}} p_{f\left(\mu^{\prime}\right) \lambda^{\prime}} h\right)<\mathcal{V}_{\mu^{\prime}}$, there exists a map $\tilde{H}: Z \times I \rightarrow X_{\lambda}$ such that $\left(\tilde{H}_{0}, p_{\lambda \lambda^{\prime}} h\right)<\mathcal{U}_{\lambda}$ and $\left(q_{\mu \mu^{\prime}} H, f_{\mu} p_{f(\mu) \lambda} \tilde{H}\right)<\mathcal{V}_{\mu}$.

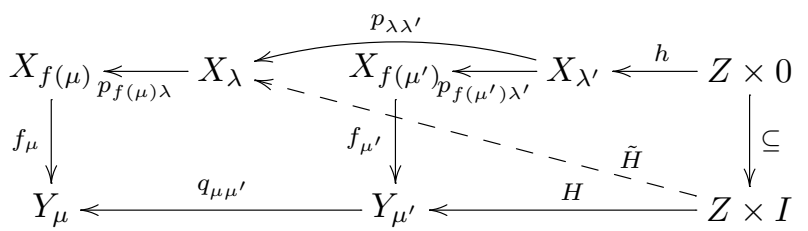

We have the following characterization of property (AHLP).

Theorem 4.1. Let $\boldsymbol{f}=\left(f, f_{\mu}\right): \mathfrak{X} \rightarrow \mathfrak{Y}$ be an approximate map, and let $s$ be a1-refinement function. Then the following implications hold.

(1.) (AHLP) for $\boldsymbol{f} \Longrightarrow(\mathrm{AHLP})^{*}$ for $s \boldsymbol{f}$, and

(2.) $(\mathrm{AHLP})^{*}$ for $s \boldsymbol{f} \Longrightarrow$ (AHLP) for $\boldsymbol{f}$.

Proof. First, to show the first assertion, assume that $f$ has property (AHLP). Let $(\lambda, \mu) \in \Lambda \times M$ be an admissible pair for $s \boldsymbol{f}$. Choose $\mu_{0} \in$ $\operatorname{AHLP}\left(s(\mu), \mathcal{V}_{s(\mu)}\right)$ for $\boldsymbol{f}$. Let $\mu^{\prime}>\mu_{0}$. Choose $\lambda_{0} \in \operatorname{AHLP}\left(s(\mu), \mathcal{V}_{s(\mu)}, \mu^{\prime}\right)$ for $f$. By (AM) there exists $\lambda^{\prime}>\lambda, \lambda_{0}$ such that

$$
\left(f_{s(\mu)} p_{f s(\mu) \lambda^{\prime}}, q_{s(\mu) \mu^{\prime}} f_{\mu^{\prime}} p_{f\left(\mu^{\prime}\right) \lambda^{\prime}}\right)<\mathcal{V}_{s(\mu)} .
$$


Choose a triple $\left(\mu_{1}, \mathcal{W}, \mu_{2}\right) \in \operatorname{AHLP}\left(s(\mu), \mathcal{V}_{s(\mu)}, \mu^{\prime}, \lambda^{\prime}\right)$ for $\boldsymbol{f}$ such that

$$
\text { st } \mathcal{V}_{\mu^{\prime \prime}}<q_{\mu_{1} \mu^{\prime \prime}}^{-1} \mathcal{W} \text { for } \mu^{\prime \prime}>\mu_{2} \text {. }
$$

Let $\mu^{\prime \prime}>\mu_{2}$. Choose $\lambda_{2} \in \operatorname{AHLP}\left(s(\mu), \mathcal{V}_{s(\mu)}, \mu^{\prime}, \lambda^{\prime}, \mu^{\prime \prime}\right)$ for $\boldsymbol{f}$. Let $\lambda^{\prime \prime}>$ $\lambda_{2}, f s\left(\mu^{\prime \prime}\right)$ such that

$$
\left(f_{\mu^{\prime \prime}} p_{f\left(\mu^{\prime \prime}\right) \lambda^{\prime \prime}}, q_{\mu^{\prime \prime} s\left(\mu^{\prime \prime}\right)} f_{s\left(\mu^{\prime \prime}\right)} p_{f s\left(\mu^{\prime \prime}\right) \lambda^{\prime \prime}}\right)<\mathcal{V}_{\mu^{\prime \prime}} .
$$

The admissible pair $\left(\lambda^{\prime \prime}, \mu^{\prime \prime}\right)$ thus defined satisfies condition (AHLP)* for $s \boldsymbol{f}$. Indeed, suppose that $h: Z \times 0 \rightarrow X_{\lambda^{\prime \prime}}$ and $H: Z \times I \rightarrow Y_{\mu^{\prime \prime}}$ are maps such that

$$
\left(H_{0}, q_{\mu^{\prime \prime} s\left(\mu^{\prime \prime}\right)} f_{s\left(\mu^{\prime \prime}\right)} p_{f s\left(\mu^{\prime \prime}\right) \lambda^{\prime \prime}} h\right)<\mathcal{V}_{\mu^{\prime \prime}}
$$

By (4.2), (4.3), (4.4),

$$
\left(q_{\mu_{1} \mu^{\prime \prime}} H_{0}, q_{\mu_{1} \mu^{\prime \prime}} f_{\mu^{\prime \prime}} p_{f\left(\mu^{\prime \prime}\right) \lambda^{\prime \prime}} h\right)<\mathcal{W} .
$$

This together with property (AHLP) implies that there exists a map $H^{\prime}$ : $Z \times I \rightarrow X_{\lambda^{\prime}}$ such that

$$
\begin{gathered}
\left(p_{\lambda^{\prime} \lambda^{\prime \prime}} h, H_{0}^{\prime}\right)<\mathcal{U}_{\lambda^{\prime}}, \\
\left(q_{s(\mu) \mu^{\prime}} f_{\mu^{\prime}} p_{f\left(\mu^{\prime}\right) \lambda^{\prime}} H^{\prime}, q_{s(\mu) \mu^{\prime \prime}} H\right)<\mathcal{V}_{s(\mu)} .
\end{gathered}
$$

By (4.5) and (AI2),

$$
\left(p_{\lambda \lambda^{\prime \prime}} h, p_{\lambda \lambda^{\prime}} H^{\prime}\right)<\mathcal{U}_{\lambda}
$$

By (4.1) and (4.6),

$$
\left(f_{s(\mu)} p_{f s(\mu) \lambda^{\prime}} H^{\prime}, q_{s(\mu) \mu^{\prime \prime}} H\right)<\operatorname{st} \mathcal{V}_{s(\mu)} .
$$

(4.8) and the fact that $s$ is a 1-refinement function imply

$$
\left(q_{\mu s(\mu)} f_{s(\mu)} p_{f s(\mu) \lambda^{\prime}} H^{\prime}, q_{\mu \mu^{\prime \prime}} H\right)<\mathcal{V}_{\mu} .
$$

By (4.7) and (4.9), the map $\tilde{H}=p_{\lambda \lambda^{\prime}} H^{\prime}: Z \times I \rightarrow X_{\lambda}$ is the desired map in $(\mathrm{AHLP})^{*}$ for $s \boldsymbol{f}$.

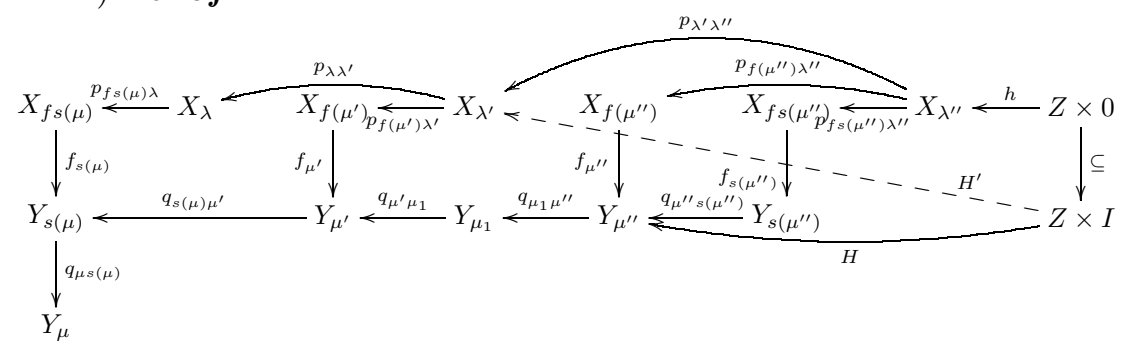

To show the second assertion, assume (AHLP)* for $s \boldsymbol{f}$. Let $\mu \in M$ and $\mathcal{V} \in \operatorname{Cov}\left(Y_{\mu}\right)$. Choose $\mu_{0}>\mu$ such that

$$
\mathcal{V}_{\mu^{\prime}}<q_{\mu \mu^{\prime}}^{-1} \mathcal{V} \text { for } \mu^{\prime}>\mu_{0}
$$


Let $\mu^{\prime}>\mu_{0}$, let $\lambda^{\prime}>\lambda_{0}=f s\left(\mu^{\prime}\right)$ and $\mathcal{U} \in \operatorname{Cov}\left(X_{\lambda^{\prime}}\right)$. Choose $\lambda_{0}^{\prime}>\lambda^{\prime}$ such that

$$
\mathcal{U}_{\lambda_{0}^{\prime}}<p_{\lambda^{\prime} \lambda_{0}^{\prime}}^{-1} \mathcal{U}
$$

Apply property $(\mathrm{AHLP})^{*}$ for $s \boldsymbol{f}$ to the admissible pair $\left(\lambda_{0}^{\prime}, \mu^{\prime}\right)$, and obtain an admissible pair $\left(\lambda_{1}^{\prime}, \mu_{1}^{\prime}\right)>\left(\lambda_{0}^{\prime}, \mu^{\prime}\right)$ that satisfies the condition in (AHLP)*. Let $\mu^{\prime \prime}>\mu_{2}=\mu_{1}=s\left(\mu_{1}^{\prime}\right)$. Choose $\lambda_{2}>\lambda_{1}^{\prime}, f\left(\mu^{\prime \prime}\right), \lambda_{0}^{\prime}$ such that

$$
\left(f_{s\left(\mu_{1}^{\prime}\right)} p_{f s\left(\mu_{1}^{\prime}\right) \lambda^{\prime \prime}}, q_{s\left(\mu_{1}^{\prime}\right) \mu^{\prime \prime}} f_{\mu^{\prime \prime}} p_{f\left(\mu^{\prime \prime}\right) \lambda^{\prime \prime}}\right)<\mathcal{V}_{s\left(\mu_{1}^{\prime}\right)} \text { for } \lambda^{\prime \prime}>\lambda_{2} .
$$

Let $\lambda^{\prime \prime}>\lambda_{2}$. To verify property (AHLP) for $\boldsymbol{f}$, suppose that $h: Z \times 0 \rightarrow X_{\lambda^{\prime \prime}}$ and $H: Z \times I \rightarrow Y_{\mu^{\prime \prime}}$ are maps such that

$$
\left(q_{\mu_{1}^{\prime} \mu^{\prime \prime}} H_{0}, q_{\mu_{1}^{\prime} \mu^{\prime \prime}} f_{\mu^{\prime \prime}} p_{f\left(\mu^{\prime \prime}\right) \lambda^{\prime \prime}} h\right)<\mathcal{V}_{\mu_{1}} .
$$

By (4.12), (4.13), and the fact that $s$ is a 1-refinement function,

$$
\left(q_{\mu_{1}^{\prime} \mu^{\prime \prime}} H_{0}, q_{\mu_{1}^{\prime} s\left(\mu_{1}^{\prime}\right)} f_{s\left(\mu_{1}^{\prime}\right)} p_{f s\left(\mu_{1}^{\prime}\right) \lambda^{\prime \prime}} h\right)<\mathcal{V}_{\mu_{1}^{\prime}} .
$$

By $(4.14)$ and $(\mathrm{AHLP})^{*}$ for $s \boldsymbol{f}$, there exists a map $H^{\prime}: Z \times I \rightarrow X_{\lambda_{0}^{\prime}}$ such that

$$
\begin{aligned}
\left(p_{\lambda_{0}^{\prime} \lambda^{\prime \prime}} h, H_{0}^{\prime}\right) & <\mathcal{U}_{\lambda_{0}^{\prime}}, \\
\left(q_{\mu^{\prime} s\left(\mu^{\prime}\right)} f_{s\left(\mu^{\prime}\right)} p_{f s\left(\mu^{\prime}\right) \lambda_{0}^{\prime}} H^{\prime}, q_{\mu^{\prime} \mu^{\prime \prime}} H\right) & <\mathcal{V}_{\mu^{\prime}} .
\end{aligned}
$$

By (4.11) and (4.15),

$$
\left(p_{\lambda^{\prime} \lambda^{\prime \prime}} h, p_{\lambda^{\prime} \lambda_{0}^{\prime}} H^{\prime}\right)<\mathcal{U}
$$

By (4.10) and (4.16),

$$
\left(q_{\mu s\left(\mu^{\prime}\right)} f_{s\left(\mu^{\prime}\right)} p_{f s\left(\mu^{\prime}\right) \lambda_{0}^{\prime}} H^{\prime}, q_{\mu \mu^{\prime \prime}} H\right)<\mathcal{V} .
$$

By (4.17) and (4.18), the map $\tilde{H}=p_{\lambda^{\prime} \lambda_{0}^{\prime}} H^{\prime}: Z \times I \rightarrow X_{\lambda^{\prime}}$ has the desired property (AHLP) for $\boldsymbol{f}$.

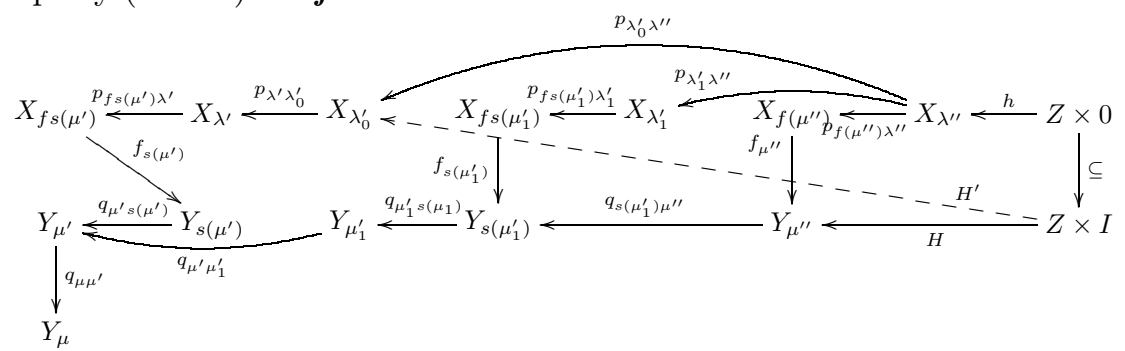

An approximate map $\boldsymbol{f}=\left(f, f_{\mu}\right): \mathfrak{X} \rightarrow \mathfrak{Y}$ is called an approximate level map provided $\Lambda=M, f=1_{\Lambda}: \Lambda \rightarrow \Lambda$, and it satisfies the following condition.

(AML) $\left(f_{\lambda} p_{\lambda \lambda^{\prime}}, q_{\lambda \lambda^{\prime}} f_{\lambda^{\prime}}\right)<\mathcal{V}_{\lambda}$ for $\lambda<\lambda^{\prime}$. 


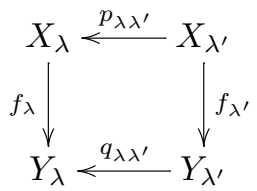

In this case we write $\left(f_{\lambda}\right)$ for $\left(f, f_{\mu}\right)$. The notion of approximate level map was first introduced in [12] under the name of special approximative map.

Every approximate map is represented by an approximate level map in the following sense [12, Theorem 2.15].

TheOREM 4.2. Let $\mathfrak{X}=\left(X_{\lambda}, \mathcal{U}_{\lambda}, p_{\lambda \lambda^{\prime}}, \Lambda\right)$ and $\mathfrak{Y}=\left(Y_{\mu}, \mathcal{V}_{\mu}, q_{\mu \mu^{\prime}}, M\right)$ be approximate systems of spaces in $\mathcal{C}$, and let $\boldsymbol{f}=\left(f, f_{\mu}\right): \mathfrak{X} \rightarrow \mathfrak{Y}$ be an approximate map. Then there exist an approximate level map $\boldsymbol{f}^{\prime}=\left(f_{\nu}^{\prime}\right): \mathfrak{X}^{\prime} \rightarrow \mathfrak{Y}^{\prime}$ between approximate systems $\mathfrak{X}^{\prime}=\left(X_{\nu}^{\prime}, \mathcal{U}_{\nu}^{\prime}, p_{\nu \nu^{\prime}}^{\prime}, N\right)$ and $\mathfrak{Y}^{\prime}=\left(Y_{\nu}^{\prime}, \mathcal{V}_{\nu}^{\prime}, q_{\nu \nu^{\prime}}^{\prime}, N\right)$ over the same index set $N$, and approximate maps $s: \mathfrak{X} \rightarrow \mathfrak{X}^{\prime}$ and $\boldsymbol{t}: \mathfrak{Y} \rightarrow \mathfrak{Y}^{\prime}$ with the following properties.

(1.) The following diagram commutes in $\mathrm{APRO}-\mathcal{C}$.

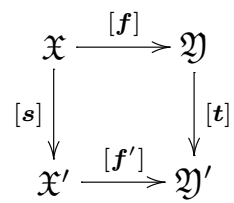

(2.) $[\boldsymbol{s}]$ and $[\boldsymbol{t}]$ are isomorphisms in APRO- $\mathcal{C}$.

(3.) $X_{\nu}^{\prime}, \mathcal{U}_{\nu}^{\prime}, p_{\nu \nu^{\prime}}^{\prime}, Y_{\nu}^{\prime}, \mathcal{V}_{\nu}^{\prime}, q_{\nu \nu^{\prime}}^{\prime}$ are some $X_{\lambda}, \mathcal{U}_{\lambda}, p_{\lambda \lambda^{\prime}}, Y_{\mu}, \mathcal{V}_{\mu}, q_{\mu \mu^{\prime}}$, respectively.

(4.) $f_{\nu}^{\prime}$ is the composite of some $p_{\lambda \lambda^{\prime}}$ and $f_{\mu}$.

Finally, in this section we discuss the AHLP for approximate level maps. In particular, we obtain a simpler condition that is equivalent to (AHLP).

For any approximate level map $\boldsymbol{f}=\left(f_{\lambda}\right): \mathfrak{X} \rightarrow \mathfrak{Y}$ and for any space $Z$, consider the following condition.

$(\mathrm{AHLP})_{L}(\forall \lambda \in \Lambda)\left(\forall \mathcal{V} \in \operatorname{Cov}\left(Y_{\lambda}\right)\right)\left(\exists \lambda_{0}>\lambda\right)\left(\forall \lambda^{\prime}>\lambda_{0}\right)(\forall \mathcal{U} \in$ $\left.\operatorname{Cov}\left(X_{\lambda^{\prime}}\right)\right)\left(\exists \lambda_{1}>\lambda^{\prime}\right)\left(\exists \mathcal{W} \in \operatorname{Cov}\left(Y_{\lambda_{1}}\right)\right)\left(\exists \lambda_{2}>\lambda_{1}\right)$ $\left(\forall \lambda^{\prime \prime}>\lambda_{2}\right)\left(\forall h: Z \times 0 \rightarrow X_{\lambda^{\prime \prime}}\right)\left(\forall H: Z \times I \rightarrow Y_{\lambda^{\prime \prime}}\right)$ $\left(q_{\lambda_{1} \lambda^{\prime \prime}} f_{\lambda^{\prime \prime}} h, q_{\lambda_{1} \lambda^{\prime \prime}} H\right)<\mathcal{W} \Longrightarrow\left(\exists \tilde{H}: Z \times I \rightarrow X_{\lambda^{\prime}}\right)$ $\left(p_{\lambda^{\prime} \lambda^{\prime \prime}} h, \tilde{H}_{0}\right)<\mathcal{U},\left(q_{\lambda \lambda^{\prime}} f_{\lambda^{\prime}} \tilde{H}, q_{\lambda \lambda^{\prime \prime}} H\right)<\mathcal{V}$

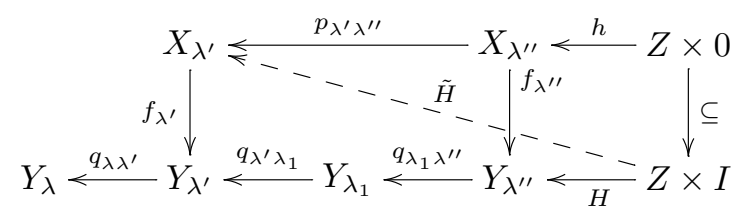


For any $\lambda \in \Lambda$ and $\mathcal{V} \in \operatorname{Cov}\left(Y_{\lambda}\right)$, let $\operatorname{AHLP}_{L}(\lambda, \mathcal{V})$ denote the set of all $\lambda_{0} \in \Lambda$ such that $\lambda_{0}>\lambda$ and $\lambda_{0}$ satisfies the condition in (AHLP) $)_{L}$. For any $\lambda \in \Lambda, \mathcal{V} \in \operatorname{Cov}\left(Y_{\lambda}\right), \lambda^{\prime}>\lambda_{0}$ where $\lambda_{0} \in \operatorname{AHLP}(\lambda, \mathcal{V})$, and $\mathcal{U} \in \operatorname{Cov}\left(X_{\lambda^{\prime}}\right)$, let $\operatorname{AHLP}\left(\lambda, \mathcal{V}, \lambda^{\prime}, \mathcal{U}\right)$ denote the set of all triples $\left(\lambda_{1}, \mathcal{W}, \lambda_{2}\right) \in \Lambda \times \operatorname{Cov}\left(Y_{\lambda_{1}}\right) \times \Lambda$ such that $\lambda_{2}>\lambda_{1}>\lambda^{\prime}$ and $\lambda_{1}, \mathcal{W}, \lambda_{2}$ satisfy condition $(\mathrm{AHLP})_{L}$.

THEOREM 4.3. An approximate level map $\boldsymbol{f}=\left(f_{\lambda}\right): \mathfrak{X} \rightarrow \mathfrak{Y}$ has property $(A H L P)$ with respect to a space $Z$ if and only if it has property $(A H L P)_{L}$ with respect to $Z$.

Proof. Suppose that an approximate level map $\boldsymbol{f}=\left(f_{\lambda}\right): \mathfrak{X} \rightarrow \mathfrak{Y}$ has property (AHLP) with respect to a space $Z$. Let $\mu \in \Lambda$ and $\mathcal{V} \in$ $\operatorname{Cov}\left(Y_{\mu}\right)$. Choose $\mu_{0} \in \operatorname{AHLP}(\lambda, \mathcal{V})$, and let $\mu^{\prime}>\mu_{0}$, and $\mathcal{U} \in \operatorname{Cov}\left(X_{\mu^{\prime}}\right)$. Choose $\lambda_{0} \in \operatorname{AHLP}\left(\mu, \mathcal{V}, \mu^{\prime}\right)$, and let $\lambda^{\prime}>\lambda_{0}$. Then choose $\left(\mu_{1}, \mathcal{W}, \mu_{2}\right) \in$ $\operatorname{AHLP}\left(\mu, \mathcal{V}, \mu^{\prime}, \lambda^{\prime}, p_{\mu^{\prime} \lambda^{\prime}}^{-1} \mathcal{U}\right)$ such that

$$
\mathcal{V}_{\mu^{\prime \prime}}<q_{\mu_{1} \mu^{\prime \prime}}^{-1} \mathcal{W}^{\prime} \text { for } \mu^{\prime \prime}>\mu_{2},
$$

where $\mathcal{W}^{\prime} \in \operatorname{Cov}\left(Y_{\mu_{1}}\right)$ is such that

$$
\text { st } \mathcal{W}^{\prime}<\mathcal{W}
$$

Let $\mu^{\prime \prime}>\mu_{2}$. Choose $\lambda_{2} \in \operatorname{AHLP}\left(\mu, \mathcal{V}, \mu^{\prime}, \lambda^{\prime}, p_{\mu^{\prime} \lambda^{\prime}}^{-1} \mathcal{U}, \mu^{\prime \prime}\right)$, and let $\lambda^{\prime \prime}>\lambda_{2}$. To verify condition $(\mathrm{AHLP})_{L}$, suppose that $h: Z \times 0 \rightarrow X_{\lambda^{\prime \prime}}$ and $H: Z \times I \rightarrow Y_{\lambda^{\prime \prime}}$ are maps such that

$$
\left(q_{\mu_{1} \lambda^{\prime \prime}} f_{\lambda^{\prime \prime}} h, q_{\mu_{1} \lambda^{\prime \prime}} H\right)<\mathcal{W}^{\prime}
$$

By (AML),

$$
\left(f_{\mu^{\prime \prime}} p_{\mu^{\prime \prime} \lambda^{\prime \prime}}, q_{\mu^{\prime \prime} \lambda^{\prime \prime}} f_{\lambda^{\prime \prime}}\right)<\mathcal{V}_{\mu^{\prime \prime}}
$$

By (4.19), (4.20), (4.21), (4.22),

$$
\left(q_{\mu_{1} \mu^{\prime \prime}} f_{\mu^{\prime \prime}} p_{\mu^{\prime \prime} \lambda^{\prime \prime}} h, q_{\mu_{1} \lambda^{\prime \prime}} H\right)<\mathcal{W} .
$$

This together with (AHLP) implies that there exists a map $H^{\prime}: Z \times I \rightarrow X_{\lambda^{\prime}}$ such that

$$
\begin{array}{r}
\left(p_{\lambda^{\prime} \lambda^{\prime \prime}} h, H_{0}^{\prime}\right)<p_{\mu^{\prime} \lambda^{\prime}}^{-1} \mathcal{U}, \\
\left(q_{\mu \mu^{\prime}} f_{\mu^{\prime}} p_{\mu^{\prime} \lambda^{\prime}} H^{\prime}, q_{\mu \lambda^{\prime \prime}} H\right)<\mathcal{V} .
\end{array}
$$

Thus the map $\tilde{H}=p_{\mu^{\prime} \lambda^{\prime}} H^{\prime}: Z \times I \rightarrow X_{\mu^{\prime}}$ is the desired map, and hence $\boldsymbol{f}$ satisfies the condition in $(\mathrm{AHLP})_{L}$.

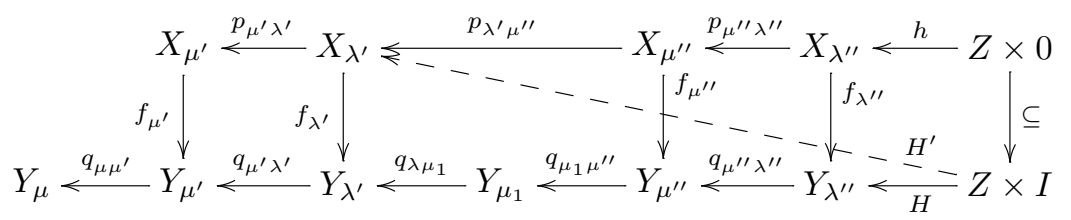


Conversely, suppose that $\boldsymbol{f}$ has property $(\mathrm{AHLP})_{L}$ with respect to a space $Z$. Let $\mu \in \Lambda$ and $\mathcal{V} \in \operatorname{Cov}\left(Y_{\mu}\right)$. Let $\mathcal{V}^{\prime} \in \operatorname{Cov}\left(Y_{\mu}\right)$ be such that

$$
\text { st } \mathcal{V}^{\prime}<\mathcal{V} \text {. }
$$

Choose $\mu_{0} \in \operatorname{AHLP}_{L}\left(\mu, \mathcal{V}^{\prime}\right)$ such that

$$
\mathcal{V}_{\mu^{\prime}}<q_{\mu \mu^{\prime}}^{-1} \mathcal{V}^{\prime} \text { for } \mu^{\prime}>\mu_{0}
$$

Let $\mu^{\prime}>\mu_{0}$, and let $\lambda^{\prime}>\lambda_{0}=\mu^{\prime}$ and $\mathcal{U} \in \operatorname{Cov}\left(X_{\lambda^{\prime}}\right)$. Choose a triple $\left(\mu_{1}, \mathcal{W}, \mu_{2}\right) \in \operatorname{AHLP}_{L}\left(\mu, \mathcal{V}, \lambda^{\prime}\right)$. Let $\mu^{\prime \prime}>\mu_{2}$, and let $\lambda^{\prime \prime}>\lambda_{2}=\mu^{\prime \prime}$. Suppose that $h: Z \times 0 \rightarrow X_{\lambda^{\prime \prime}}$ and $H: Z \times I \rightarrow Y_{\lambda^{\prime \prime}}$ are maps such that

$$
\left(q_{\mu_{1} \lambda^{\prime \prime}} f_{\lambda^{\prime \prime}} h, q_{\mu_{1} \lambda^{\prime \prime}} H\right)<\mathcal{W} .
$$

Then by $(\mathrm{AHLP})_{L}$, there exists a map $\tilde{H}: Z \times I \rightarrow X_{\lambda^{\prime}}$ such that

$$
\begin{aligned}
\left(p_{\lambda^{\prime} \lambda^{\prime \prime}} h, \tilde{H}_{0}\right) & <\mathcal{U}, \\
\left(q_{\mu \lambda^{\prime}} f_{\lambda^{\prime}} \tilde{H}, q_{\mu \lambda^{\prime \prime}} H\right) & <\mathcal{V}^{\prime} .
\end{aligned}
$$

By (AML),

$$
\left(f_{\mu^{\prime}} p_{\mu^{\prime} \lambda^{\prime}}, q_{\mu^{\prime} \lambda^{\prime}} f_{\lambda^{\prime}}\right)<\mathcal{V}_{\mu^{\prime}}
$$

By (4.23), (4.24), (4.26), and (4.27),

$$
\left(q_{\mu \mu^{\prime}} f_{\mu^{\prime}} p_{\mu^{\prime} \lambda^{\prime}} \tilde{H}, q_{\mu \lambda^{\prime \prime}} H\right)<\mathcal{V} .
$$

(4.25) and (4.28) show that $\boldsymbol{f}$ has property (AHLP) as required.

\section{Composition AXIOM}

In this section we discuss the composition axiom for a fibration category in the sense of Baues [1].

Theorem 5.1. Let $\mathfrak{X}=\left(X_{\lambda}, \mathcal{U}_{\lambda}, p_{\lambda \lambda^{\prime}}, \Lambda\right), \mathfrak{Y}=\left(Y_{\mu}, \mathcal{V}_{\mu}, q_{\mu \mu^{\prime}}, M\right), \mathfrak{Z}=$ $\left(Z_{\nu}, \mathcal{W}_{\nu}, r_{\nu \nu^{\prime}}, N\right)$ be approximate systems in $\mathcal{C}$. Let $\boldsymbol{f}=\left(f, f_{\mu}\right): \mathfrak{X} \rightarrow \mathfrak{Y}$ be an approximate map, and let $\boldsymbol{g}=\left(g, g_{\nu}\right): \mathfrak{Y} \rightarrow \mathfrak{Z}$ be a uniform approximate map. If $\boldsymbol{f}$ and $\boldsymbol{g}$ have the AHLP with respect to a space $Z$, then so does the composite $s(\boldsymbol{g} \boldsymbol{f}): \mathfrak{X} \rightarrow \mathfrak{Y}$.

Proof. Let $\nu \in N$ and $\mathcal{W} \in \operatorname{Cov}\left(Z_{\nu}\right)$. Take $\mathcal{W}^{\prime} \in \operatorname{Cov}\left(Z_{\nu}\right)$ such that

$$
\text { st } \mathcal{W}^{\prime}<\mathcal{W} \text {. }
$$

Since $\boldsymbol{g}$ has property (AHLP), then $\boldsymbol{s} \boldsymbol{g}$ has property (AHLP). Choose $\nu_{0} \in$ $\operatorname{AHLP}\left(\nu, \mathcal{W}^{\prime}\right)$ for $s \boldsymbol{g}$ such that

$$
\mathcal{W}_{\nu^{\prime}}<r_{\nu \nu^{\prime}}^{-1} \mathcal{W}^{\prime} \text { for } \nu^{\prime}>\nu_{0}
$$

and let $\nu^{\prime}>\nu_{0}$. Choose $\mu_{0} \in M$ such that $\mu_{0} \in \operatorname{AHLP}\left(g s\left(\nu^{\prime}\right), g_{s\left(\nu^{\prime}\right)}^{-1} r_{\nu s\left(\nu^{\prime}\right)}^{-1} \mathcal{W}^{\prime}\right)$ for $\boldsymbol{f}$ and $\mu_{0} \in \operatorname{AHLP}\left(\nu, \mathcal{W}^{\prime}, \nu^{\prime}\right)$ for $s \boldsymbol{g}$, and let $\mu^{\prime}>\mu_{0}$. Choose $\lambda_{0} \in$ $\operatorname{AHLP}\left(g s\left(\nu^{\prime}\right), g_{s\left(\nu^{\prime}\right)}^{-1} r_{\nu s\left(\nu^{\prime}\right)}^{-1} \mathcal{W}^{\prime}, \mu^{\prime}\right)$ for $\boldsymbol{f}$ such that $\lambda_{0}>f\left(\mu^{\prime}\right), f g s\left(\nu^{\prime}\right)$ and

$$
\left(f_{g s\left(\nu^{\prime}\right)} p_{f g s\left(\nu^{\prime}\right) \lambda^{\prime}}, q_{g s\left(\nu^{\prime}\right) \mu^{\prime}} f_{\mu^{\prime}} p_{f\left(\mu^{\prime}\right) \lambda^{\prime}}\right)<\mathcal{V}_{s g\left(\nu^{\prime}\right)} \text { for } \lambda^{\prime}>\lambda_{0}
$$


and let $\lambda^{\prime}>\lambda_{0}$, and $\mathcal{U} \in \operatorname{Cov}\left(X_{\lambda^{\prime}}\right)$. Then we can choose a triple $\left(\mu_{1}, \mathcal{V}, \mu_{2}\right) \in$ $\operatorname{AHLP}\left(g s\left(\nu^{\prime}\right), g_{s\left(\nu^{\prime}\right)}^{-1} r_{\nu s\left(\nu^{\prime}\right)}^{-1} \mathcal{W}^{\prime}, \mu^{\prime}, \lambda^{\prime}, \mathcal{U}\right)$ for $\boldsymbol{f}$ such that

$$
\mathcal{V}_{\mu^{\prime \prime}}<q_{\mu_{1} \mu^{\prime \prime}}^{-1} \mathcal{V}^{\prime} \text { for } \mu^{\prime \prime}>\mu_{2}
$$

where $\mathcal{V}^{\prime} \in \operatorname{Cov}\left(Y_{\mu_{1}}\right)$ satisfies

$$
\text { st } \mathcal{V}^{\prime}<\mathcal{V}
$$

Let $\mu^{\prime \prime}>\mu_{2}$. Choose $\lambda_{2} \in \operatorname{AHLP}\left(g s\left(\nu^{\prime}\right), g_{s\left(\nu^{\prime}\right)}^{-1} r_{\nu s\left(\nu^{\prime}\right)}^{-1} \mathcal{W}^{\prime}, \mu^{\prime}, \lambda^{\prime}, \mathcal{U}, \mu^{\prime \prime}\right)$ for $\boldsymbol{f}$, and choose a triple $\left(\nu_{1}, \mathcal{W}_{1}, \nu_{2}\right) \in \operatorname{AHLP}\left(\nu, \mathcal{W}^{\prime}, \nu^{\prime}, \mu^{\prime \prime}, q_{\mu_{1} \mu^{\prime \prime}}^{-1} \mathcal{V}^{\prime}\right)$ for $s \boldsymbol{g}$ such that

$$
\mathcal{W}_{\nu^{\prime \prime}}<r_{\nu_{1} \nu^{\prime \prime}}^{-1} \mathcal{W}_{1}^{\prime} \text { for } \nu^{\prime \prime}>\nu_{2}
$$

where $\mathcal{W}_{1}^{\prime} \in \operatorname{Cov}\left(Y_{\nu_{1}}\right)$ satisfies

$$
\text { st } \mathcal{W}_{1}^{\prime}<\mathcal{W}_{1}
$$

Let $\nu^{\prime \prime}>\nu_{2}$. Choose $\mu_{3} \in \operatorname{AHLP}\left(\nu, \mathcal{W}^{\prime}, \nu^{\prime}, \mu^{\prime \prime}, q_{\mu_{1} \mu^{\prime \prime}}^{-1} \mathcal{V}^{\prime}, \nu^{\prime \prime}\right)$ for $s \boldsymbol{g}$, and let $\mu>\mu_{3}$. Let $\lambda^{\prime \prime}>f g s\left(\nu^{\prime \prime}\right), f\left(\mu^{\prime \prime}\right), f(\mu), \lambda_{2}$ such that

$$
\begin{array}{r}
\left(f_{\mu^{\prime \prime}} p_{f\left(\mu^{\prime \prime}\right) \lambda^{\prime \prime}}, q_{\mu^{\prime \prime} \mu} f_{\mu} p_{f(\mu) \lambda^{\prime \prime}}\right)<\mathcal{V}_{\mu^{\prime \prime}}, \\
\left(f_{g s\left(\nu^{\prime \prime}\right)} p_{f g s\left(\nu^{\prime \prime}\right) \lambda^{\prime \prime}}, q_{g s\left(\nu^{\prime \prime}\right) \mu} f_{\mu} p_{f(\mu) \lambda^{\prime \prime}}\right)<\mathcal{V}_{g s\left(\nu^{\prime \prime}\right)} .
\end{array}
$$

To verify property (AHLP) for $s(\boldsymbol{g} \boldsymbol{f})$, suppose that $h: Z \times 0 \rightarrow X_{\lambda^{\prime \prime}}$ and $H: Z \times I \rightarrow Y_{\nu^{\prime \prime}}$ are maps such that

$$
\left(r_{\nu_{1} s\left(\nu^{\prime \prime}\right)} g_{s\left(\nu^{\prime \prime}\right)} f_{g s\left(\nu^{\prime \prime}\right)} p_{f g s\left(\nu^{\prime \prime}\right) \lambda^{\prime \prime}} h, r_{\nu_{1} \nu^{\prime \prime}} H_{0}\right)<\mathcal{W}_{1}^{\prime}
$$

By (5.3), (5.6), (5.7), and the assumption that $\boldsymbol{g}$ is uniform,

$$
\left(r_{\nu_{1} \nu^{\prime \prime}} g_{s\left(\nu^{\prime \prime}\right)} q_{g s\left(\nu^{\prime \prime}\right) \mu} f_{\mu} p_{f(\mu) \lambda^{\prime \prime}}, r_{\nu_{1} s\left(\nu^{\prime \prime}\right)} H_{0}\right)<\mathcal{W}_{1} .
$$

By (5.11) and (AHLP) for $s \boldsymbol{g}$, there exists a map $\tilde{H}: Z \times I \rightarrow Y_{\mu^{\prime \prime}}$ such that

$$
\begin{array}{r}
\left(q_{\mu^{\prime \prime} \mu} f_{\mu} p_{f(\mu) \lambda^{\prime \prime}} h, \tilde{H}_{0}\right)<q_{\mu_{1} \mu^{\prime \prime}}^{-1} \mathcal{V}^{\prime}, \\
\left(r_{\nu s\left(\nu^{\prime}\right)} g_{s\left(\nu^{\prime}\right)} q_{g s\left(\nu^{\prime}\right) \mu^{\prime \prime}} \tilde{H}, r_{\nu \nu^{\prime \prime}} H\right)<\mathcal{W}^{\prime} .
\end{array}
$$

By (5.4), (5.5), (5.8), and (5.12),

$$
\left(q_{\mu_{1} \mu^{\prime \prime}} f_{\mu^{\prime \prime}} p_{f\left(\mu^{\prime \prime}\right) \lambda^{\prime \prime}} h, q_{\mu_{1} \mu^{\prime \prime}} \tilde{H}_{0}\right)<\mathcal{V} .
$$

By (AHLP) for $f$ and (5.14), there exists a map $K: Z \times I \rightarrow X_{\lambda^{\prime}}$ such that

$$
\begin{array}{r}
\left(p_{\lambda^{\prime} \lambda^{\prime \prime}} h, K_{0}\right)<\mathcal{U}, \\
\left(q_{g s\left(\nu^{\prime}\right) \mu^{\prime}} f_{\mu^{\prime}} p_{f\left(\mu^{\prime}\right) \lambda^{\prime}} K, q_{g s\left(\nu^{\prime}\right) \mu^{\prime \prime}} \tilde{H}\right)<g_{s\left(\nu^{\prime}\right)}^{-1} r_{\nu s\left(\nu^{\prime}\right)}^{-1} \mathcal{W}^{\prime} .
\end{array}
$$

By (5.2), (5.3), and the assumption that $\boldsymbol{g}$ is uniform,

(5.17) $\quad\left(r_{\nu s\left(\nu^{\prime}\right)} g_{s\left(\nu^{\prime}\right)} f_{g s\left(\nu^{\prime}\right)} p_{f g s\left(\nu^{\prime}\right) \lambda^{\prime}}, r_{\nu s\left(\nu^{\prime}\right)} g_{s\left(\nu^{\prime}\right)} q_{g s\left(\nu^{\prime}\right) \mu^{\prime}} f_{\mu^{\prime}} p_{f\left(\mu^{\prime}\right) \lambda^{\prime}}\right)<\mathcal{W}^{\prime}$.

By (5.1), (5.13), (5.16) and (5.17),

$$
\left(r_{\nu s\left(\nu^{\prime}\right)} g_{s\left(\nu^{\prime}\right)} f_{g s\left(\nu^{\prime}\right)} p_{f g s\left(\nu^{\prime}\right) \lambda^{\prime}} K, r_{\nu^{\prime \prime}} H\right)<\mathcal{W} .
$$


By (5.15) and (5.18), we conclude that $s(\boldsymbol{g} \boldsymbol{f})$ has the AHLP.

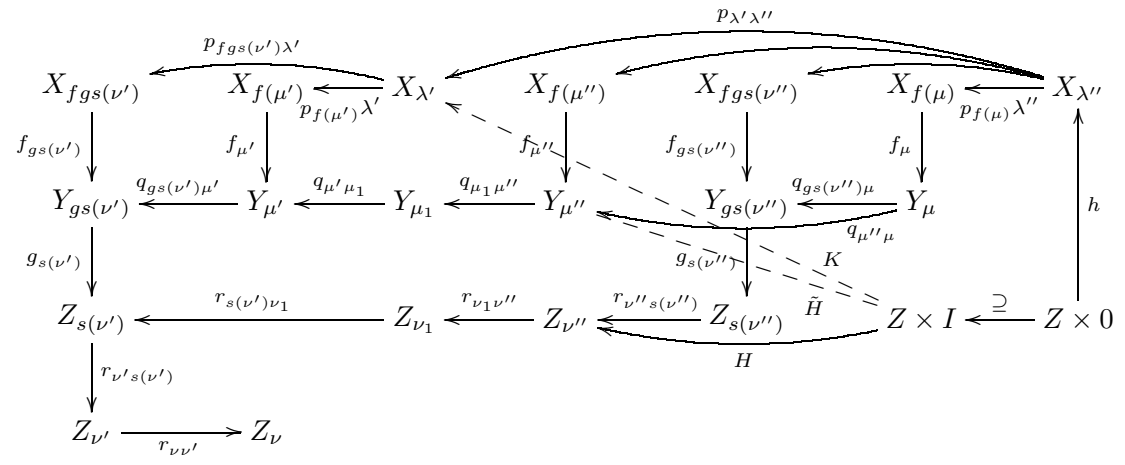

Theorem 5.2. Let $\mathfrak{X}=\left(X_{\lambda}, \mathcal{U}_{\lambda}, p_{\lambda \lambda^{\prime}}, \Lambda\right)$ and $\mathfrak{Y}=\left(Y_{\mu}, \mathcal{V}_{\mu}, q_{\mu \mu^{\prime}}, M\right)$ be approximate systems in $\mathcal{C}$, and let $\boldsymbol{f}=\left(f, f_{\mu}\right): \mathfrak{X} \rightarrow \mathfrak{Y}$ be an approximate map. If $\boldsymbol{f}$ represents an isomorphism in $\mathrm{APRO}-\mathcal{C}$, then $\boldsymbol{f}$ has the AHLP with respect to any space.

Proof. Let $\mu \in M$ and $\mathcal{V} \in \operatorname{Cov}\left(Y_{\mu}\right)$. Take $\mathcal{V}^{\prime} \in \operatorname{Cov}\left(Y_{\mu}\right)$ such that

$$
\text { st } \mathcal{V}^{\prime}<\mathcal{V}
$$

Choose $\mu_{0}>\mu$ such that

$$
\mathcal{V}_{\mu^{\prime}}<q_{\mu \mu^{\prime}}^{-1} \mathcal{V}^{\prime} \text { for } \mu^{\prime}>\mu_{0}
$$

Let $\mu^{\prime}>\mu_{0}$, let $\lambda^{\prime}>\lambda_{0}=f\left(\mu^{\prime}\right)$, and let $\mathcal{U} \in \operatorname{Cov}\left(X_{\lambda^{\prime}}\right)$. Take $\lambda_{0}^{\prime}>\lambda^{\prime}$ such that

$$
\operatorname{st} \mathcal{U}_{\lambda_{0}^{\prime}}<p_{\lambda^{\prime} \lambda_{0}^{\prime}}^{-1} \mathcal{U}
$$

By Theorem 2.6, there exist $\mu_{1}>\mu^{\prime}, \lambda_{1}>f\left(\mu_{1}\right), \lambda_{0}^{\prime}$, and a map $k: Y_{\mu_{1}} \rightarrow X_{\lambda_{0}^{\prime}}$ such that

$$
\begin{array}{r}
\left(k f_{\mu_{1}} p_{f\left(\mu_{1}\right) \lambda_{1}}, p_{\lambda_{0}^{\prime} \lambda_{1}}\right)<\mathcal{U}_{\lambda_{0}^{\prime}}, \\
\mathcal{V}_{\mu_{1}}<k^{-1} \mathcal{U}_{\lambda_{0}^{\prime}}, \\
\left(f_{\mu^{\prime}} p_{f\left(\mu^{\prime}\right) \lambda_{0}^{\prime}} k, q_{\mu^{\prime} \mu_{1}}\right)<\operatorname{st} \mathcal{V}_{\mu^{\prime}}
\end{array}
$$

Set $\mu_{2}=\mu_{1}$, and let $\mu^{\prime \prime}>\mu_{2}$. By (AM) there is $\lambda_{2}>\lambda_{1}, f\left(\mu^{\prime \prime}\right)$ such that

$$
\left(f_{\mu_{1}} p_{f\left(\mu_{1}\right) \lambda^{\prime \prime}}, q_{\mu_{1} \mu^{\prime \prime}} f_{\mu^{\prime \prime}} p_{f\left(\mu^{\prime \prime}\right) \lambda^{\prime \prime}}\right)<\mathcal{V}_{\mu_{1}} \text { for } \lambda^{\prime \prime}>\lambda_{2} .
$$

Let $\lambda^{\prime \prime}>\lambda_{2}$. Suppose that $h: Z \times 0 \rightarrow X_{\lambda^{\prime \prime}}$ and $H: Z \times I \rightarrow Y_{\mu^{\prime \prime}}$ are maps such that

$$
\left(q_{\mu_{1} \mu^{\prime \prime}} f_{\mu^{\prime \prime}} p_{f\left(\mu^{\prime \prime}\right) \lambda^{\prime \prime}} h, q_{\mu_{1} \mu^{\prime \prime}} H\right)<\mathcal{V}_{\mu_{1}}
$$


Let $\tilde{H}=p_{\lambda^{\prime} \lambda_{0}^{\prime}} k q_{\mu_{1} \mu^{\prime \prime}} H: Z \times I \rightarrow X_{\lambda^{\prime}}$. By (5.21), (5.22), (5.23), (5.25), and (5.26), we have

$$
\left(p_{\lambda^{\prime} \lambda^{\prime \prime}} h, \tilde{H}\right)<\mathcal{U} .
$$

By (5.19), (5.20), and (5.24), we have

$$
\left(q_{\mu \mu^{\prime}} f_{\mu^{\prime}} p_{f\left(\mu^{\prime}\right) \lambda^{\prime}} \tilde{H}, q_{\mu \mu^{\prime \prime}} H\right)<\mathcal{V} .
$$

By (5.27) and (5.28) we see that $\tilde{H}$ has the required property, showing that $f$ has the AHLP with respect to $Z$.

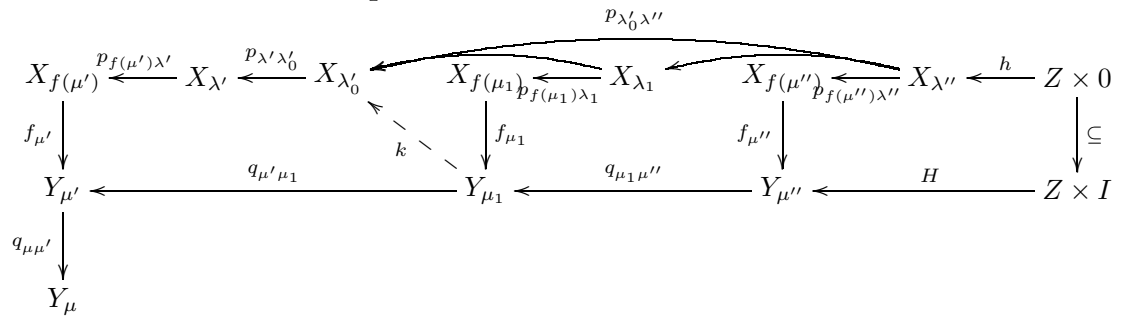

For any approximate map $\boldsymbol{f}=\left(f, f_{\mu}\right): \mathfrak{X} \rightarrow \mathfrak{Y}$ between approximate systems $\mathfrak{X}=\left(X_{\lambda}, \mathcal{U}_{\lambda}, p_{\lambda \lambda^{\prime}}, \Lambda\right)$ and $\mathfrak{Y}=\left(Y_{\mu}, \mathcal{V}_{\mu}, q_{\mu \mu^{\prime}}, M\right)$, where $\mathfrak{Y}$ has the following property

(H) any two $\mathcal{V}_{\mu}$-near maps into $Y_{\mu}$ are homotopic,

there is an induced system map $\mathrm{H}(\boldsymbol{f}): \mathrm{H}(\mathfrak{X}) \rightarrow \mathrm{H}(\mathfrak{Y})$ between the systems $\mathrm{H}(\mathfrak{X})=\left(X_{\lambda}, \mathrm{H}\left(p_{\lambda \lambda^{\prime}}\right), \Lambda\right)$ and $\mathrm{H}(\mathfrak{Y})=\left(Y_{\mu}, \mathrm{H}\left(q_{\mu \mu^{\prime}}\right), M\right)$ in the pro-homotopy category pro- $\mathrm{H}(\mathcal{C})$. Here, for any map $f: X \rightarrow Y, \mathrm{H}(f)$ denotes the homotopy class of $f$.

Note that for any approximate maps $\boldsymbol{f}=\left(f, f_{\mu}\right), \boldsymbol{g}=\left(g, g_{\mu}\right): \mathfrak{X} \rightarrow \mathfrak{Y}$, $\boldsymbol{f} \sim \boldsymbol{g}$ implies that for each $\mu \in M$ there exists $\lambda>f(\mu), g(\mu)$ such that $\mathrm{H}\left(f_{\mu}\right) \mathrm{H}\left(p_{f(\mu) \lambda}\right)=\mathrm{H}\left(g_{\mu}\right) \mathrm{H}\left(p_{g(\mu) \lambda}\right)$. Thus, for each approximate map $\boldsymbol{f}: \mathfrak{X} \rightarrow$ $\mathfrak{Y}$, there is a well-defined morphism $\mathrm{H}([\boldsymbol{f}]): \mathrm{H}(\mathfrak{X}) \rightarrow \mathrm{H}(\mathfrak{Y})$ in pro- $\mathrm{H}(\mathcal{C})$.

A fibration category in the sense of Baues is a category with two classes of morphisms singled out, the class fib of morphisms called fibrations and the class we of morphisms called weak equivalences, subject to four axioms (F1)-(F4). The first one, called the composition axiom, asserts that

(F1) Isomorphisms are fibrations and weak equivalences. If two of the morphisms $f: X \rightarrow Y, g: Y \rightarrow Z$, and $g f: X \rightarrow Z$ are weak equivalences, then so is the third. Moreover, the composition of fibrations is a fibration.

For more details, the reader is referred to [1].

Theorems 5.1 and 5.2 immediately imply the following corollary.

COROLLARY 5.3. The full subcategory of APRO- $\mathcal{C}$, whose objects are approximate systems with property (H), fibrations are morphisms having the 
AHLP with respect to arbitrary space, and weak equivalences are morphisms which induce isomorphisms in pro- $\mathrm{H}(\mathcal{C})$, satisfy the composition axiom (F1).

Corollary 5.4. For any $[\boldsymbol{f}] \in E(\boldsymbol{p}, \boldsymbol{q})$ and $\left[\boldsymbol{f}^{\prime}\right] \in E\left(\boldsymbol{p}^{\prime}, \boldsymbol{q}^{\prime}\right)$ where $\boldsymbol{p}, \boldsymbol{p}^{\prime} \in$ $E(X)$ and $\boldsymbol{q}, \boldsymbol{q}^{\prime} \in E(Y)$, if $[\boldsymbol{f}] \equiv_{a}\left[\boldsymbol{f}^{\prime}\right]$ and if $[\boldsymbol{f}]$ has the AHLP with respect to a space $Z$, so does $\left[\boldsymbol{f}^{\prime}\right]$.

Proof. If $[\boldsymbol{f}] \equiv_{a}\left[\boldsymbol{f}^{\prime}\right]$, then $\left[\boldsymbol{f}^{\prime}\right]=\left[1_{Y}\right]_{\boldsymbol{q}, \boldsymbol{q}^{\prime}}[\boldsymbol{f}]\left[1_{X}\right]_{\boldsymbol{p}^{\prime}, \boldsymbol{p}}$. Theorem 2.5 implies that $\left[1_{X}\right]_{\boldsymbol{p}^{\prime}, \boldsymbol{p}}$ and $\left[1_{Y}\right]_{\boldsymbol{q}, \boldsymbol{q}^{\prime}}$ are isomorphisms in APRO-APol, and hence by Theorem 5.2 they have the AHLP with respect to any space. This fact together with Theorem 5.1 and the assumption that $[\boldsymbol{f}]$ has the AHLP with respect to a space $Z$ implies that $\left[f^{\prime}\right]$ has the AHLP with respect to $Z$.

Thus the AHLP is well-defined for morphisms in ASh. A morphism $F \in$ $\operatorname{ASh}(X, Y)$ is said to have the approximate homotopy lifting property (AHLP) with respect to a space $Z$ provided there is a representative $[\boldsymbol{f}] \in E(\boldsymbol{p}, \boldsymbol{q})$ of $F$ which has the AHLP with respect to $Z$. This is equivalent to saying that any representative of $F$ has the AHLP with respect to $Z$.

Let Sh denote the shape category [7]. There is a functor $A S S: \mathrm{ASh} \rightarrow \mathrm{Sh}$ which is defined as follows. For each object $X$ in ASh, let $A S S(X)=X$. If $F: X \rightarrow Y$ is a morphism in ASh, by Theorem 2.4 and Lemma 5.5 below, $F$ is represented by an approximate map $\boldsymbol{f}: \mathfrak{X} \rightarrow \mathfrak{Y}$ between approximate systems $\mathfrak{X}$ and $\mathfrak{Y}$ with property $(\mathrm{H})$ for some approximate Pol-resolutions. Then $\boldsymbol{f}: \mathfrak{X} \rightarrow \mathfrak{Y}$ induces a system map $\mathrm{H}(\boldsymbol{f}): \mathrm{H}(\mathfrak{X}) \rightarrow \mathrm{H}(\mathfrak{Y})$. Let $A S S(F)$ be the morphism in Sh which is represented by $\mathrm{H}(\boldsymbol{f})$.

LEMmA 5.5. ([12, 5.2]) Every space $X$ admits an approximate Polresolution $\boldsymbol{p}: X \rightarrow \mathfrak{X}$ with property $(H)$.

COROLlary 5.6. The category ASh together with fibrations defined as morphisms having the AHLP with respect to arbitrary space and weak equivalences defined as morphisms, which induce isomorphisms in Sh, satisfies the composition axiom (F1).

Proof. Let $\langle[\boldsymbol{f}]\rangle \in \operatorname{ASh}(X, Y)$ and $\langle[\boldsymbol{g}]\rangle \in \operatorname{ASh}(Y, Z)$ have the AHLP with respect to a space $Z$. Let $\boldsymbol{f}: \mathfrak{X} \rightarrow \mathfrak{Y}$ and $\boldsymbol{g}: \mathfrak{Y}^{\prime} \rightarrow \mathfrak{Z}$ be approximate maps for some approximate resolutions $\boldsymbol{p}: X \rightarrow \mathfrak{X}, \boldsymbol{q}: Y \rightarrow \mathfrak{Y}, \boldsymbol{q}: Y \rightarrow \mathfrak{Y}^{\prime}$, and $\boldsymbol{r}: Z \rightarrow \mathfrak{Z}$. Then there is an approximate map $\boldsymbol{j}: \mathfrak{Y} \rightarrow \mathfrak{Y}^{\prime}$ which induces an isomorphism in APRO-APol and hence has the AHLP with respect to any space by Theorem 5.2. So, the composite $\langle[\boldsymbol{f}]\rangle \circ\langle[\boldsymbol{g}]\rangle=\langle[\boldsymbol{g}] \circ[\boldsymbol{j}] \circ[\boldsymbol{f}]\rangle$ is represented by the composite of the morphisms in APRO-APol with the AHLP, and hence it has the AHLP with respect to any space by Theorem 5.1.

If $\langle[\boldsymbol{f}]\rangle \in \operatorname{ASh}(X, Y)$ is an isomorphism, then $[\boldsymbol{f}]$ is an isomorphism in APRO-APol and hence has the AHLP with respect to any space by Theorem 5.2. So, $\langle[\boldsymbol{f}]\rangle$ has the AHLP with respect to any space. 
Finally, that for any morphisms $F \in \operatorname{ASh}(X, Y)$ and $G \in \operatorname{ASh}(Y, Z)$, any two of $F, G$, and $G \circ F$ are isomorphisms, so is the third follows from the fact that there is a functor $A S S:$ ASh $\rightarrow$ Sh.

\section{SHAPE FiBRATiOnS}

Recall that a map $f: X \rightarrow Y$ between spaces is a shape fibration provided there is a resolution $(\boldsymbol{p}, \boldsymbol{q}, \boldsymbol{f})$ of $f$ such that the system map $\boldsymbol{f}: \boldsymbol{X} \rightarrow \boldsymbol{Y}$ has the AHLP with respect to arbitrary space, where $\boldsymbol{p}: X \rightarrow \boldsymbol{X}$ and $\boldsymbol{q}: Y \rightarrow \boldsymbol{Y}$ are APol-resolutions of $X$ and $Y$, respectively [4].

In this section, as a consequence of the composition axiom discussed in the previous section, we characterize the shape fibration in terms of the AHLP between approximate systems and discuss its consequences.

THEOREM 6.1. A map $f: X \rightarrow Y$ between spaces is a shape fibration if and only if there is an approximate resolution $(\boldsymbol{p}, \boldsymbol{q}, \boldsymbol{f})$ of $f$, where $\boldsymbol{p}: X \rightarrow \mathfrak{X}$ and $\boldsymbol{q}: Y \rightarrow \mathfrak{Y}$ are approximate APol-resolutions such that the approximate system map $\boldsymbol{f}: \mathfrak{X} \rightarrow \mathfrak{Y}$ has the AHLP with respect to any spaces.

Before proving the theorem, we prove the following three facts.

Lemma 6.2. Let $f: X \rightarrow Y$ be a map between spaces. Suppose that $(\boldsymbol{p}, \boldsymbol{q}, \boldsymbol{f})$ and $\left(\boldsymbol{p}^{\prime}, \boldsymbol{q}^{\prime}, \boldsymbol{f}^{\prime}\right)$ are two approximate resolutions of $f$. If $\boldsymbol{f}$ has the AHLP with respect to a space $Z$, so does $\boldsymbol{f}^{\prime}$.

Proof. Let $(\boldsymbol{p}, \boldsymbol{q}, \boldsymbol{f})$ and $\left(\boldsymbol{p}^{\prime}, \boldsymbol{q}^{\prime}, \boldsymbol{f}^{\prime}\right)$ be two approximate resolutions of $f$, where $\boldsymbol{p}: X \rightarrow \mathfrak{X}, \boldsymbol{p}^{\prime}: X \rightarrow \mathfrak{X}^{\prime}, \boldsymbol{q}: Y \rightarrow \mathfrak{Y}, \boldsymbol{q}^{\prime}: Y \rightarrow \mathfrak{Y}^{\prime}$ are approximate APol-resolutions, and $f: \mathfrak{X} \rightarrow \mathfrak{Y}$ and $\boldsymbol{f}^{\prime}: \mathfrak{X}^{\prime} \rightarrow \mathfrak{Y}^{\prime}$ are approximate maps. Theorem 2.4 implies that there exist approximate maps $\boldsymbol{i}: \mathfrak{X} \rightarrow \mathfrak{X}^{\prime}$ and $\boldsymbol{j}: \mathfrak{Y} \rightarrow \mathfrak{Y}^{\prime}$ such that $\left(\boldsymbol{p}, \boldsymbol{p}^{\prime}, \boldsymbol{i}\right)$ and $\left(\boldsymbol{q}, \boldsymbol{q}^{\prime}, \boldsymbol{j}\right)$ are approximate resolutions of the identity maps $1_{X}: X \rightarrow X$ and $1_{Y}: Y \rightarrow Y$, respectively. Since $\left(\boldsymbol{p}, \boldsymbol{q}^{\prime}, s\left(\boldsymbol{f}^{\prime} \boldsymbol{i}\right)\right)$ and $\left(\boldsymbol{p}, \boldsymbol{q}^{\prime}, s(\boldsymbol{j} \boldsymbol{f})\right)$ are approximate resolutions of $f$, Theorem 2.5 implies that $s\left(\boldsymbol{f}^{\prime} \boldsymbol{i}\right) \equiv s(\boldsymbol{j} \boldsymbol{f})$, and hence $[\boldsymbol{j}] \circ[\boldsymbol{f}]=\left[\boldsymbol{f}^{\prime}\right] \circ[\boldsymbol{i}]$. Then $[\boldsymbol{i}]$ and $[\boldsymbol{j}]$ are isomorphisms in APRO-APol, and hence, by Theorems 5.1 and 5.2, the composite $\left[\boldsymbol{f}^{\prime}\right]=[\boldsymbol{j}] \circ[\boldsymbol{f}] \circ[\boldsymbol{i}]^{-1}$ and hence $\boldsymbol{f}^{\prime}$ has the AHLP with respect to any space as required.

Lemma 6.3. (1.) Every resolution $\boldsymbol{p}=\left(\boldsymbol{p}_{\lambda}\right): X \rightarrow \boldsymbol{X}=\left(X_{\lambda}, p_{\lambda \lambda^{\prime}}, \Lambda\right)$ in Top admits an approximate resolution $\overline{\boldsymbol{p}}=\left(\bar{p}_{\alpha}\right): X \rightarrow \mathfrak{X}=$ $\left(\bar{X}_{\alpha}, \mathcal{U}_{\alpha}, \bar{p}_{\alpha \alpha^{\prime}}, \bar{\Lambda}\right)$ and an increasing function $\sigma_{\boldsymbol{X}}: \bar{\Lambda} \rightarrow \Lambda$ with the following properties:

(a) $\bar{\Lambda}$ is cofinite, directed, and antisymmetric.

(b) $\bar{X}_{\alpha}=X_{\sigma_{\boldsymbol{X}}(\alpha)}$ and $\bar{p}_{\alpha}=p_{\sigma_{\boldsymbol{X}}(\alpha)}$ for $\alpha \in \bar{\Lambda}$, and $\bar{p}_{\alpha \alpha^{\prime}}=$ $p_{\sigma_{X}(\alpha) \sigma_{\boldsymbol{X}}\left(\alpha^{\prime}\right)}$ for $\alpha<\alpha^{\prime}$.

(c) $\mathcal{U}_{\alpha^{\prime}}<\bar{p}_{\alpha \alpha^{\prime}}^{-1} \mathcal{U}_{\alpha}$ for $\alpha<\alpha^{\prime}$. 
(d) for any $\lambda \in \Lambda$ and for any $\mathcal{U} \in \operatorname{Cov}\left(X_{\lambda}\right)$ there exists $\alpha \in \bar{\Lambda}$ such that $\sigma_{\boldsymbol{X}}(\alpha)=\lambda$ and $\mathcal{U}_{\alpha}=\mathcal{U}$.

(e) the system map $\left(\sigma_{\boldsymbol{X}}, 1_{\sigma_{\boldsymbol{X}}(\alpha)}\right): \boldsymbol{X} \rightarrow \overline{\boldsymbol{X}}=\left(\bar{X}_{\alpha}, \bar{p}_{\alpha \alpha^{\prime}}, \bar{\Lambda}\right)$ represents an isomorphism in pro- $\mathcal{C}$.

(2.) Every system map $\boldsymbol{f}=\left(f, f_{\mu}\right): \boldsymbol{X} \rightarrow \boldsymbol{Y}=\left(Y_{\mu}, q_{\mu \mu^{\prime}}, M\right)$ with $f$ being an increasing function admits a commutative approximate map $\bar{f}=\left(\bar{f}, \bar{f}_{\beta}\right): \mathfrak{X} \rightarrow \mathfrak{Y}=\left(\bar{Y}_{\beta}, \mathcal{V}_{\beta}, \bar{q}_{\beta \beta^{\prime}}, \bar{M}\right)$ such that

(a) $\bar{f}$ is an increasing function such that $\sigma_{\boldsymbol{X}} \bar{f}(\beta) \geq f \sigma_{\boldsymbol{Y}}(\beta)$ for $\beta \in \bar{M}$

(b) $\bar{f}_{\beta}=f_{\sigma_{\boldsymbol{Y}}(\beta)} p_{f \sigma_{\boldsymbol{Y}}(\beta), \sigma_{\boldsymbol{X}} \bar{f}(\beta)}: \bar{X}_{\bar{f}(\beta)} \rightarrow \bar{Y}_{\beta}$,

(c) $\mathcal{U}_{\bar{f}(\beta)}<\bar{f}_{\beta}^{-1} \mathcal{V}_{\beta}$ for $\beta \in \bar{M}$,

(d) the following diagram commutes for $\beta \in \bar{M}$.

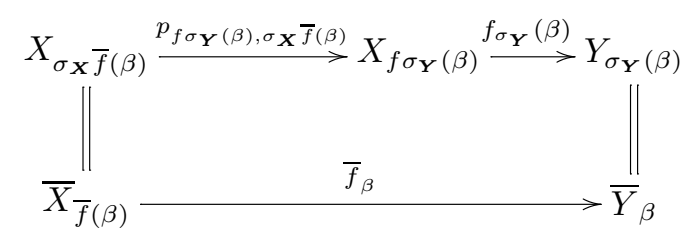

(3.) If the system map $\boldsymbol{f}$ has the AHLP with respect to a space $Z$, so does the approximate map $\overline{\boldsymbol{f}}$.

Proof. This is proven in [12, Proposition 3.7] and [10]. Note here that (3) easily follows from the definition of $\overline{\boldsymbol{f}}$.

Lemma 6.4. Every map $f: X \rightarrow Y$ admits an approximate resolution $(\boldsymbol{p}, \boldsymbol{q}, \boldsymbol{f})$ of $f$ such that $\boldsymbol{f}: \mathfrak{X} \rightarrow \mathfrak{Y}$ is a commutative approximate map between approximate systems, where $\boldsymbol{p}: X \rightarrow \mathfrak{X}$ and $\boldsymbol{q}: Y \rightarrow \mathfrak{Y}$ are approximate APolresolutions of $X$ and $Y$, respectively.

Proof. There exists a resolution $(\boldsymbol{p}, \boldsymbol{q}, \boldsymbol{f})$ of $f$ where $\boldsymbol{p}: X \rightarrow \boldsymbol{X}$ and $\boldsymbol{q}: Y \rightarrow \boldsymbol{Y}$ are APol-resolutions of $X$ and $Y$, respectively, and $\boldsymbol{f}: \boldsymbol{X} \rightarrow \boldsymbol{Y}$ is a system map whose limit is $f$ [4, Theorem 13]. By Lemma 6.3, the APolresolutions $\boldsymbol{p}: X \rightarrow \boldsymbol{X}$ and $\boldsymbol{q}: Y \rightarrow \boldsymbol{Y}$ admit approximate APol-resolutions $\overline{\boldsymbol{p}}: X \rightarrow \mathfrak{X}$ and $\overline{\boldsymbol{q}}: Y \rightarrow \mathfrak{Y}$, respectively, with the properties in Lemma 6.3 (1). The system map $\boldsymbol{f}: \boldsymbol{X} \rightarrow \boldsymbol{Y}$ admits a commutative approximate map $\overline{\boldsymbol{f}}: \mathfrak{X} \rightarrow \mathfrak{Y}$ with the properties in Lemma 6.3 (2). Thus such obtained triple $(\overline{\boldsymbol{p}}, \overline{\boldsymbol{q}}, \overline{\boldsymbol{f}})$ is an approximate resolution of $f$ as required.

Proof of Theorem 6.1. Suppose that there is an approximate resolution $(\boldsymbol{p}, \boldsymbol{q}, \boldsymbol{f})$ of $f$ such that the approximate system map (not necessarily commutative) $\boldsymbol{f}: \mathfrak{X} \rightarrow \mathfrak{Y}$ has the AHLP with respect to any spaces. By Lemma 6.4, there is an approximate resolution $\left(\boldsymbol{p}^{\prime}, \boldsymbol{q}^{\prime}, \boldsymbol{f}^{\prime}\right)$ of $f$ such that $\boldsymbol{f}^{\prime}: \mathfrak{X} \rightarrow \mathfrak{Y}$ is a commutative approximate map. By Corollary 5.4, that $\boldsymbol{f}$ has the AHLP implies that $\boldsymbol{f}^{\prime}$ has the AHLP. This fact together with Theorem 3.1 implies 
that the system map $\boldsymbol{f}^{\prime}$ which is obtained by forgetting the meshes of the approximate systems $\mathfrak{X}$ and $\mathfrak{Y}$ has the AHLP. This means that $f$ is a shape fibration.

Conversely, suppose that a map $f: X \rightarrow Y$ is a shape fibration. Then this together with Lemma 6.3 implies that there is an approximate resolution $(\boldsymbol{p}, \boldsymbol{q}, \boldsymbol{f})$ of $f$ such that $\boldsymbol{f}: \mathfrak{X} \rightarrow \mathfrak{Y}$ is a commutative approximate map and has the AHLP as required.

Corollary 6.5. A map $f: X \rightarrow Y$ between spaces is a shape fibration if and only if $A S(f)$ has the AHLP with respect to any space.

Proof. This immediately follows from Theorem 6.1 and Corollary 5.4.

As an application of the composition axiom for approximate maps, we have

THEOREM 6.6. Every homeomorphism $f: X \rightarrow Y$ between topologically complete spaces is a shape fibration.

Proof. Theorem 2.7 implies that there exists an approximate resolution $(\boldsymbol{p}, \boldsymbol{q}, \boldsymbol{f})$ of $f$ such that $\langle[\boldsymbol{f}]\rangle$ is an isomorphism in ASh. Then the approximate map $\boldsymbol{f}$ induces an isomorphism in APRO-APol, and hence, by Theorem 5.2, $f$ has the AHLP with respect to any spaces. By Theorem 6.1 we conclude that $f$ is a shape fibration.

\section{REFERENCES}

[1] H. J. Baues, Algebraic homotopy, Cambridge Univ. Press, Cambridge, 1989.

[2] Q. Haxhibeqiri, Shape fibrations for topological spaces, Glas. Mat. Ser. III 17(37) (1982), 381-401.

[3] S. Mardešić, Mappings of inverse systems, Glasnik Mat.-Fiz. Astronom. Društvo Mat. Fiz. Hrvatske Ser. II 18 (1963), 241-254.

[4] S. Mardešić, Approximate polyhedra, resolutions of maps and shape fibrations, Fund. Math. 114 (1981), 53-78.

[5] S. Mardešić and L. Rubin, Approximate inverse systems of compacta and covering dimension, Pacific J. Math. 138 (1989), 129-144.

[6] S. Mardešić and T. B. Rushing, Shape fibrations I, General Toppology Appl. 9 (1978), 193-215.

[7] S. Mardešić and J. Segal, Shape Theory, North Holland-Publishing Co., AmsterdamNew York, 1982.

[8] S. Mardešić and T. Watanabe, Approximate resolutions of spaces and maps, Glas. Mat. Ser. III 24(44) (1989), 587-637.

[9] J. Mioduszewski, Mappings of inverse limits, Colloq. Math. 10 (1963), 39-44.

[10] T. Miyata, Pull-backs and fibrations in approximate pro-categories, Glas. Mat. Ser. III 43(63) (2008), 451-479.

[11] T. Watanabe, Approximate expansions of maps into inverse systems, in: Geometric and Algebraic Topology, Banach Center Publ. 18 (1986), 363- 370.

[12] T. Watanabe, Approximative shape. I. Basic notions, Tsukuba J. Math. 11 (1987), $17-59$. 
[13] T. Yagasaki, Fiber shape theory, shape fibrations and movability of maps, Lecture Notes in Math. 1283, Springer, Berlin, 1987, 240-252.

T. Miyata

Department of Mathematics and Informatics

Graduate School of Human Development and Environment

Kobe University

Kobe, 657-8501

Japan

E-mail: tmiyata@kobe-u.ac.jp

Received: 12.12.2007.

Revised: 21.3.2008. 